\title{
HOMOGENIZATION OF THE MIXED BOUNDARY-VALUE PROBLEM FOR A FORMALLY SELFADJOINT ELLIPTIC SYSTEM IN A PERIODICALLY PUNCHED DOMAIN
}

\author{
G. CARDOnE, A. CORBO ESPOSITO, AND S. A. NAZAROV
}

\begin{abstract}
A generalized Gårding-Korn inequality is established in a domain $\Omega(h) \subset$ $\mathbb{R}^{n}$ with a small, of size $O(h)$, periodic perforation, without any restrictions on the shape of the periodicity cell, except for the usual assumptions that the boundary is Lipschitzian, which ensures the Korn inequality in a general domain. Homogenization is performed for a formally selfadjoint elliptic system of second order differential equations with the Dirichlet or Neumann conditions on the outer or inner parts of the boundary, respectively; the data of the problem are assumed to satisfy assumptions of two types: additional smoothness is required from the dependence on either the "slow" variables $x$, or the "fast" variables $y=h^{-1} x$. It is checked that the exponent $\delta \in(0,1 / 2]$ in the accuracy $O\left(h^{\delta}\right)$ of homogenization depends on the smoothness properties of the problem data.
\end{abstract}

\section{§1. Problem settings, Preliminaries, And Description of Results}

1. Boundary-value problem. Let $\varpi \subset \mathbb{R}^{n}$ be a periodicity cell, i.e., an open (not necessarily connected) set in the unit cube

$$
\mathbb{Q}=\left\{x=\left(x_{1}, \ldots, x_{n}\right): 0<x_{j}<1, j=1, \ldots, n\right\}
$$

(see Remark 1.1 below). We introduce the perforated space П (see Figure 2 and compare to Figure 1, c)) as the interior of the set

$$
\bar{\Pi}=\bigcup_{\alpha \in \mathbb{Z}^{n}} \overline{\varpi(\alpha)}
$$

where $\alpha=\left(\alpha_{1}, \ldots, \alpha_{n}\right)$ is a multiindex, $\mathbb{Z}=\{0, \pm 1, \pm 2, \ldots\}$ and the $\varpi(\alpha)=\{x: x-\alpha \in$ $\varpi\}$ are integral shifts of the cell. We assume that $\Pi$ is a domain (open connected set) bounded by an $(n-1)$-dimensional surface; the smoothness of the latter will be specified below. In any case, the boundary $\partial \Pi$ is Lipschitzian; due to the periodic structure of $\partial \Pi$, the Lipschitz property needs verification only inside a ball $\mathbb{B}_{R}=\{x:|x|<R\}$ of some radius $R>\sqrt{n}$.

Also, let $\Omega$ be a domain in the Euclidean space $\mathbb{R}^{n}$, with smooth boundary $\partial \Omega$ and compact closure $\bar{\Omega}=\Omega \cup \partial \Omega$; smoothness will be specified later. The perforated domain $\Omega(h)$ is a large (of volume $O\left(h^{0}\right)$ ) connected component of the set

$$
\Omega \cap \Pi_{h},
$$

where $h \in(0,1]$ is a small parameter, and $\Pi_{h}$ is the $h$-compression of $\Pi$, i.e.,

$$
\Pi_{h}=\left\{x: y:=h^{-1} x \in \Pi\right\} .
$$

2010 Mathematics Subject Classification. Primary 35J57.

Key words and phrases. Gårding-Korn inequality, homogenization, formally selfadjoint elliptic system, rate of convergence.

S. A. Nazarov was supported by RFBR (grant no. 09-00759). 


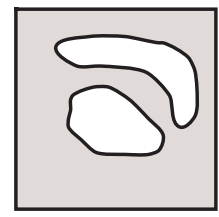

a)

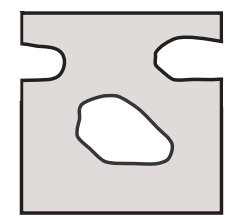

b)

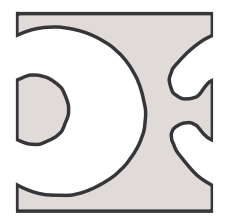

c)

\section{FIGURE 1}

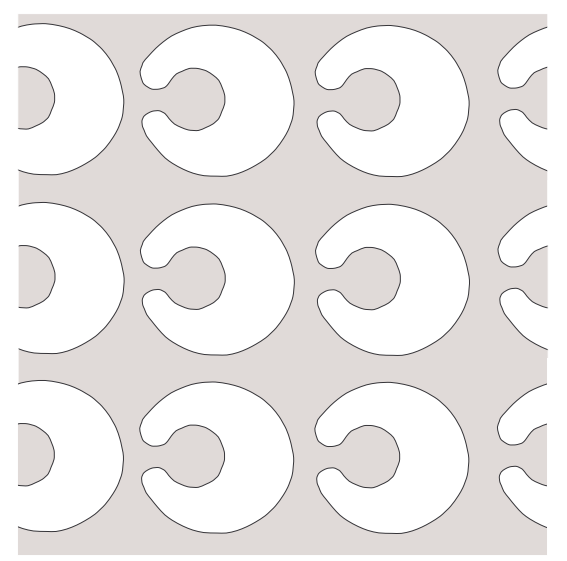

FiguRE 2

We call $y=\left(y_{1}, \ldots, y_{n}\right)$ the fast variables, or the dilated coordinates. The boundary $\partial \Omega$ splits into two subsets, its outer and inner parts

$$
\Gamma(h)=\partial \Omega \cap \Pi_{h} \quad \text { and } \quad \Upsilon(h)=\Omega \cap \partial \Pi_{h} .
$$

In the perforated domain introduced above, we consider the following mixed boundaryvalue problem:

$$
\begin{aligned}
\mathcal{L}^{h}\left(x, \nabla_{x}\right) u^{h}(x) & =f^{h}(x), & & x \in \Omega(h), \\
\mathcal{N}^{h}\left(x, \nabla_{x}\right) u^{h}(x) & =g^{h}(x), & & x \in \Upsilon(h), \\
u^{h}(x) & =0, & & x \in \Gamma(h),
\end{aligned}
$$

where $u^{h}=\left(u_{1}^{h}, \ldots, u_{k}^{h}\right)^{\top}$ and $f^{h}=\left(f_{1}^{h}, \ldots, f_{k}^{h}\right)^{\top}, g^{h}=\left(g_{1}^{h}, \ldots, g_{k}^{h}\right)^{\top}$ are vector-valued functions written as columns (i.e., $T$ means transposition; in particular, it would be correct to write $x=\left(x_{1}, \ldots, x_{n}\right)^{\top}$ in (1.1)). The dependence of the right-hand sides of problem (1.6)-(1.8) on the parameter $h$ will be specified later; finding the asymptotics of its solution $u^{h}$ as $h \rightarrow+0$ is one of the main goals in this paper.

The matrices $\mathcal{L}^{h}$ and $\mathcal{N}^{h}$ of differential operators of the second and first order (respectively), which occur on the left-hand sides in (1.6) and (1.7), need a detailed description. In the formula

$$
\mathcal{L}^{h}\left(x, \nabla_{x}\right)=\mathcal{D}\left(\nabla_{x}\right)^{*} \mathcal{A}\left(x, h^{-1} x\right) \mathcal{D}\left(\nabla_{x}\right),
$$

the matrix-valued function $(x, y) \mapsto \mathcal{A}(x, y)$ of size $N \times N$ depends both on the slow variables $x \in \Omega$ and on the fast variables $y \in \varpi$, and the latter dependence is 1-periodic in $y_{1}, \ldots, y_{n}$. Precise smoothness conditions will be imposed later, but the entries of $\mathcal{A}$ will always be bounded and measurable, and for a.e. $(x, y) \in \Omega \times \varpi$ the matrix $\mathcal{A}(x, y)$ 
itself will be Hermitian and positive definite with the eigenvalues uniformly separated away from zero. The differential operator $\mathcal{D}\left(\nabla_{x}\right)^{*}={\overline{\mathcal{D}\left(-\nabla_{x}\right)}}^{\top}$ is formally adjoint to the $(N \times k)$-matrix $\mathcal{D}\left(\nabla_{x}\right)$ of first order differential operators with constant complex coefficients (the bar means complex conjugation). Also, $\nabla_{x}=\left(\partial / \partial x_{1}, \ldots, \partial / \partial x_{n}\right)^{\top}$ is the gradient operator, $\mathcal{D}(0)=0 \in \mathbb{C}^{N \times k}$, and

$$
\mathcal{N}^{h}\left(x, \nabla_{x}\right)={\overline{\mathcal{D}}\left(\nu^{h}(x)\right)^{\top}}^{\top} \mathcal{A}\left(x, h^{-1} x\right) \mathcal{D}\left(\nabla_{x}\right),
$$

where $\nu^{h}=\left(\nu_{1}^{h}, \ldots, \nu_{n}^{h}\right)^{\top}$ is the unit vector (column) of the outward normal to the surface $\Upsilon(h)$ (see the definition (1.5)).

Since $\mathcal{N}^{h}$ is the Neumann boundary condition operator, in the case where the data are not smooth enough we should interpret problem (1.6)-(1.8) as the integral identity

$$
\begin{aligned}
a\left(u^{h}, v^{h} ; \Omega(h)\right): & =\left(\mathcal{A D}\left(\nabla_{x}\right) u^{h}, \mathcal{D}\left(\nabla_{x}\right) v^{h}\right)_{\Omega(h)} \\
& =\left(f^{h}, v^{h}\right)_{\Omega(h)}+\left(g^{h}, v^{h}\right)_{\Upsilon(h)}, \quad v^{h} \in \stackrel{\circ}{H}^{1}(\Omega(h) ; \Gamma(h))^{k},
\end{aligned}
$$

and deal with its weak solution $u^{h} \in \stackrel{\circ}{H}^{1}(\Omega(h) ; \Gamma(h))^{k}$. Here $\stackrel{\circ}{H}^{1}(\Omega(h) ; \Gamma(h))^{k}$ is the space of vector-valued functions satisfying the Dirichlet condition (1.8), and the upper index $k$ is absent in the notation of scalar products and norms; e.g., in identity (1.11), by $(,)_{\Xi}$ we mean the natural scalar product in the Lebesgue space $L_{2}(\Xi)$, scalar or vector, of any dimension. Under the minimal restrictions on $\mathcal{A}$ and $\Upsilon(h)$ described above, all terms in the integral identity (1.11) (and, in particular, the Hermitian sesquilinear form $a$ ) are well defined for any $f^{h} \in L_{2}(\Omega(h))^{k}, g^{h} \in L_{2}(\Upsilon(h))^{k}$.

We assume that the matrix $\mathcal{D}(\xi)$ is algebraically complete (see [2, $\S 3.4$, p. 191]), i.e., that there is a positive integer $\varrho_{\mathcal{D}} \in \mathbb{N}:=\{1,2, \ldots\}$ such that for any row $p(\xi)=$ $\left(p_{1}(\xi), \ldots, p_{k}(\xi)\right)$ of homogeneous polynomials of degree $\varrho \geq \varrho_{\mathcal{D}}$ in the variables $\xi=$ $\left(\xi_{1}, \ldots, \xi_{n}\right)^{\top}$ there exists a polynomial row $q(\xi)=\left(q_{1}(\xi), \ldots, q_{N}(\xi)\right)$ satisfying

$$
p(\xi)=q(\xi) \mathcal{D}(\xi), \quad \xi \in \mathbb{R}^{n} .
$$

The algebraic completeness of $\mathcal{D}$ and the positive definiteness of $\mathcal{A}$ ensure the polynomial property [3, 4] of the form $a$, namely,

$$
a(u, u ; \Xi)=0,\left.u \in H^{1}(\Xi)^{k} \Longleftrightarrow u \in \mathcal{P}\right|_{\Xi} .
$$

Here $\Xi$ is an arbitrary subdomain in $\Omega(h)$, and $\mathcal{P}$ is some finite-dimensional linear space of vector polynomials $x=\left(x_{1}, \ldots, x_{n}\right)^{\top}$. By [3, 4, we have

$$
\mathcal{P}=\left\{p=\left(p_{1}, \ldots, p_{k}\right)^{\top}: \mathcal{D}\left(\nabla_{x}\right) p(x)=0 \in \mathbb{C}^{N}, x \in \mathbb{R}^{n}\right\},
$$

and the degrees of the scalar polynomials $p_{j}$ forming the column $p$ on the right in (1.14) do not exceed $\varrho_{\mathcal{D}}-1$. We put $d=\operatorname{dim} \mathcal{P}$, introduce a basis $\mathbf{p}^{1}, \ldots, \mathbf{p}^{d}$ in $\mathcal{P}$, and construct the following polynomial matrix of size $k \times d$ :

$$
\mathbf{p}(x)=\left(\mathbf{p}^{1}(x), \ldots, \mathbf{p}^{d}(x)\right) .
$$

Since the linear coordinate changes $x \mapsto \gamma x$ and $x \mapsto x-x^{0}$, where $\gamma>0$ and $x^{0} \in \mathbb{R}^{n}$, do not affect the linear space (1.14), we see that any derivative of a vector polynomial $p \in \mathcal{P}$, as well as the homogeneous terms, stay in $\mathcal{P}$. Thus, we can choose the basis elements $\mathbf{p}^{1}, \ldots, \mathbf{p}^{d}$ to be homogeneous polynomials such that

$$
0=\operatorname{deg} \mathbf{p}^{1}=\cdots=\operatorname{deg} \mathbf{p}^{n}<\operatorname{deg} \mathbf{p}^{n+1} \leq \cdots \leq \operatorname{deg} \mathbf{p}^{d}
$$

and $\mathbf{p}^{p}(x)=e_{p}=\left(\delta_{p, 1}, \ldots, \delta_{p, n}\right)^{\top}$ is the unit vector of the $x_{p}$-axis $\left(\delta_{p, q}\right.$ is the Kronecker symbol) 1

\footnotetext{
${ }^{1}$ In Subsection 3 of $\S 2$ we shall need another basis.
} 


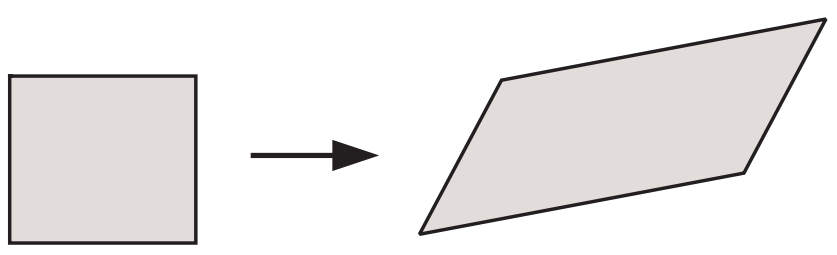

Figure 3

The general results of 2, 3, 4, show that, under our assumptions about the matrices $\mathcal{A}$ and $\mathcal{D}$, the operator (1.9) becomes formally positive and elliptic, and the operator (1.10) of the Neumann boundary conditions covers $\mathcal{L}^{h}$ on the inner boundary $\Upsilon(h)$; i.e., the Shapiro-Lopatinski conditions are fulfilled. The fact that $\mathcal{L}^{h}$ is covered by the Dirichlet conditions on the outer boundary $\Gamma(h)$ is obvious.

Remark 1.1. Any affine transformation $x \mapsto x^{\prime}=m x$ with a nonsingular numerical real $(n \times n)$-matrix $m$ transforms the cube (1.1) to an $n$-dimensional skew parallelepiped, keeping the properties of $\mathcal{A}$ and $\mathcal{D}$ mentioned above. Thus, the assumption that the perforated space $\Pi$ has cubic structure is not too restrictive.

2. Specific systems of differential equations. In the survey [4, the reader can find a series of mathematical physics problems that fall under the requirements listed in Subsection 1 of $\S 1$; here we restrict ourselves to discussing three of them.

Example 1.1. Suppose $k=1, N=n$, and $\mathcal{D}\left(\nabla_{x}\right)=\nabla_{x}$. In this case, $\mathcal{L}^{h}\left(x, \nabla_{x}\right)=$ $-\nabla_{x}^{\top} \mathcal{A}\left(x, h^{-1} x\right) \nabla_{x}$ is a scalar operator in the divergence form, and $\varrho_{\mathcal{D}}=1, \mathcal{P}=\mathbb{C}$.

Example 1.2. For the 3 -dimensional $(n=3)$ linearized system of elasticity theory that describes the deformation of an anisotropic and nonhomogeneous composite body, the operator $\mathcal{L}^{h}$, written in the matrix (rather than tensor; see [5, 6]) form, is composed of a rapidly oscillating matrix $\mathcal{A}\left(x, h^{-1} x\right)$ of elastic modules and a matrix $\mathcal{D}$ of size $6 \times 3$,

$$
\mathcal{D}(\xi)^{\top}=\left(\begin{array}{cccccc}
\xi_{1} & 0 & 0 & 0 & 2^{-1 / 2} \xi_{3} & 2^{1 / 2} \xi_{2} \\
0 & \xi_{2} & 0 & 2^{-1 / 2} \xi_{3} & 0 & 2^{-1 / 2} \xi_{1} \\
0 & 0 & \xi_{3} & 2^{-1 / 2} \xi_{2} & 2^{-1 / 2} \xi_{1} & 0
\end{array}\right) .
$$

It is not hard to check that the matrix (1.12) is algebraically complete (see (1.17); in particular, $k=3, N=6$, and $\varrho_{\mathcal{D}}=2$. As to the polynomial property (1.13), it involves the linear space

$$
\mathcal{P}=\left\{p(x)=d(x) a: a=\left(a_{1}, a_{2}, a_{3}, a_{4}, a_{5}, a_{6}\right)^{\top} \in \mathbb{R}^{6}\right\},
$$

where $d(x)$ is a $(3 \times 6)$-matrix depending linearly on the variables $x_{j}$, namely,

$$
d(x)=\left(\begin{array}{cccccc}
1 & 0 & 0 & 0 & 2^{-1 / 2} x_{3} & -2^{1 / 2} x_{2} \\
0 & 1 & 0 & -2^{-1 / 2} x_{3} & 0 & 2^{-1 / 2} x_{1} \\
0 & 0 & 1 & 2^{-1 / 2} x_{2} & -2^{-1 / 2} x_{1} & 0
\end{array}\right) .
$$

The vectors $d(x) a$ are rigid-body displacements, translational $\left(a_{4}=a_{5}=a_{6}=0\right)$ or rotational $\left(a_{1}=a_{2}=a_{3}=0\right)$. Due to the factors $2^{-1 / 2}$, the column of deformations

$$
\mathcal{D}\left(\nabla_{x}\right) u=\left(\varepsilon_{11}(u), \varepsilon_{22}(u), \varepsilon_{33}(u), 2^{1 / 2} \varepsilon_{23}(u), 2^{1 / 2} \varepsilon_{31}(u), 2^{1 / 2} \varepsilon_{12}(u)\right)^{\top}
$$

(see [6, Chapter 2]) has the same natural norm as the deformation tensor with the Cartesian coordinates

$$
\varepsilon_{j k}(u)=\frac{1}{2}\left(\frac{\partial u_{j}}{\partial x_{k}}+\frac{\partial u_{k}}{\partial x_{j}}\right) .
$$


The column $\mathcal{A D}\left(\nabla_{x}\right) u$ has a structure similar to (1.18) and contains components of the stress tensor. The matrix $\mathcal{A}$, which relates the columns of stresses and deformations, is called the stiffness matrix, or the Hook matrix; its symmetry and positive definiteness are prescribed by the nature of the elastic medium.

Example 1.3. Suppose $n=3, k=4, N=9$,

$$
\mathcal{D}(\xi)^{\top}=\left(\begin{array}{ccccccccc}
\xi_{1} & 0 & 0 & 0 & 2^{-1 / 2} \xi_{3} & 2^{-1 / 2} \xi_{2} & 0 & 0 & 0 \\
0 & \xi_{2} & 0 & 2^{-1 / 2} \xi_{3} & 0 & 2^{-1 / 2} \xi_{1} & 0 & 0 & 0 \\
0 & 0 & \xi_{3} & 2^{-1 / 2} \xi_{2} & 2^{-1 / 2} \xi_{1} & 0 & 0 & 0 & 0 \\
0 & 0 & 0 & 0 & 0 & 0 & \xi_{1} & \xi_{2} & \xi_{3}
\end{array}\right),
$$

and the matrix $\mathcal{A}$ is of the form

$$
\mathcal{A}=\left(\begin{array}{cc}
\mathcal{A}_{(1,1)} & \mathcal{A}_{(1,2)} \\
\mathcal{A}_{(2,1)} & -\mathcal{A}_{(2,2)}
\end{array}\right)
$$

where $\mathcal{A}_{(1,1)}$ and $\mathcal{A}_{(2,2)}$ are positive definite matrix-valued functions of size $6 \times 6$ and $3 \times 3$, respectively, and $\mathcal{A}_{(1,2)}=\mathcal{A}_{(2,1)}^{\top}$ is a matrix-valued function of size $6 \times 3$. The differential operator serves for a description of a piezoelectric medium with rapidly oscillating properties: the first three elements of the column $u$ are rigid-body displacements, and the fourth is the electric potential (see, e.g., the monograph [7). The matrix (1.19) is obtained by adding the row $\left(0,0,0,0,0,0, \xi_{1}, \xi_{2}, \xi_{3}\right)$ to the $(3 \times 6)$-matrix (1.17) (also, the row of nine zeros is added above it); therefore, the matrix (1.19) satisfies (1.13). However, the matrix (1.20) does not meet the above conditions, at least because of the "wrong" sign in the right lower block; in particular, the operator (1.6) fails to be formally positive. Nevertheless, in [4. Example 1.13] and in [8] it was shown how to reduce the spectral problem to a form suitable for analysis by methods of the present paper.

3. Solvability of the mixed boundary-value problem. The most important consequence of the fact that the matrix $\mathcal{D}$ is algebraically complete is the following generalized Korn inequality, established in [2, Theorem 7.3.6, p. 191]:

$$
\left\|\nabla_{x} u ; L_{2}(\Xi)\right\|^{2} \leq c_{\Xi}\left(\left\|\mathcal{D}\left(\nabla_{x}\right) u ; L_{2}(\Xi)\right\|^{2}+\left\|u ; L_{2}(\Xi)\right\|^{2}\right),
$$

where $u \in H^{1}(\Xi)^{k}$, and $\Xi$ is a bounded domain in $\mathbb{R}^{n}$ with a Lipschitzian boundary.

As has already been mentioned, the inner boundary $\Upsilon(h)$ is assumed to be Lipschitzian, and in the domain $\Omega(h)$ we have the generalized Gårding-Korn inequality

$$
\left\|u ; H^{1}(\Omega(h))\right\|^{2} \leq c(h)\left\|\mathcal{D}\left(\nabla_{x}\right) u ; L_{2}(\Omega(h))\right\|^{2}, \quad u \in \stackrel{\circ}{H}^{1}(\Omega(h) ; \Gamma(h))^{k},
$$

which is deduced from (1.21) with the use of the following two observations.

Lemma 1.1 (on equivalent norms). If $\Xi$ is a domain with Lipschitzian boundary and compact closure, then

$$
\left\|u ; H^{1}(\Xi)\right\|^{2} \leq c_{\Xi, \mathcal{F}}\left(\left\|\mathcal{D}\left(\nabla_{x}\right) u ; L_{2}(\Xi)\right\|^{2}+|\mathcal{F}(u)|^{2}\right), \quad u \in H^{1}(\Xi)^{k},
$$

where $\mathcal{F}: H^{1}(\Xi)^{k} \rightarrow \mathbb{C}^{m}$ is a functional with the following properties:

$$
\mathcal{F}(t u)=t \mathcal{F}(u), \quad t>0
$$

$$
\begin{gathered}
u^{j} \rightarrow u^{\infty} \text { weakly in } H^{1}(\Xi)^{k} \Longrightarrow \mathcal{F}\left(u^{j}\right) \rightarrow \mathcal{F}\left(u^{\infty}\right) \text { in } \mathbb{C}^{m} \text { as } j \rightarrow+\infty ; \\
\mathcal{F}(p)=0 \in \mathbb{C}^{m}, \quad p \in \mathcal{P} \Longrightarrow p=0 .
\end{gathered}
$$

The factor $c_{\Xi, \mathcal{F}}$ is independent of $u \in H^{1}(\Xi)^{k}$.

Proof. This statement is well known; we include the proof for the reader's convenience.

By inequality (1.21), it suffices to verify the relation

$$
\left\|u ; L_{2}(\Xi)\right\|^{2} \leq c\left(\left\|\mathcal{D}\left(\nabla_{x}\right) u ; L_{2}(\Xi)\right\|^{2}+|\mathcal{F}(u)|^{2}\right) .
$$


Suppose this inequality fails; then there is a sequence $\left\{u^{j}\right\}$ in $H^{1}(\Xi)^{k}$ such that

$$
\left\|u^{j} ; L_{2}(\Xi)\right\|=1, \quad\left\|\mathcal{D}\left(\nabla_{x}\right) u^{j} ; L_{2}(\Xi)\right\| \rightarrow 0, \quad\left|\mathcal{F}\left(u^{j}\right)\right| \rightarrow 0 .
$$

Using (1.21), we see that the norms $\left\|\nabla_{x} u^{j} ; L_{2}(\Xi)\right\|$ are uniformly bounded; then there exists a subsequence $\left\{u^{j_{q}}\right\}$ that converges to a vector-valued function $u^{\infty} \in H^{1}(\Xi)^{k}$ weakly in $H^{1}(\Xi)^{k}$ and strongly in $L_{2}(\Xi)^{k}$. Since $\mathcal{D}\left(\nabla_{x}\right) u^{j_{q}} \rightarrow 0$ strongly in $L_{2}(\Xi)^{N}$, we have $\mathcal{D}\left(\nabla_{x}\right) u^{\infty}=0$; the polynomial property ensures that $u^{\infty} \in \mathcal{P}$. Also, we have $\mathcal{F}\left(u^{j_{q}}\right) \rightarrow 0=\mathcal{F}\left(u^{\infty}\right)$, i.e., $u^{\infty}=0$ by the last property in (1.24), but this contradicts the first formula in (1.25).

Lemma 1.2. Let $\Xi$ be the same as in Lemma 1.1, and let $\Sigma$ denote either the domain $\Xi$ itself, or its boundary $\partial \Xi$. Then for any $u \in H^{1}(\Xi)^{k}$ we have

$$
\left\|u ; H^{1}(\Xi)\right\|^{2} \leq c_{\Xi, \Sigma}\left(\left\|\mathcal{D}\left(\nabla_{x}\right) u ; L_{2}(\Xi)\right\|^{2}+\left|\int_{\Sigma} \overline{\mathbf{p}}(x)^{\top} u(x) d s_{x}\right|^{2}\right) .
$$

Here $d s_{x}=d x$ in the case where $\Sigma=\Xi$, and $d s_{x}$ is the $(n-1)$-dimensional surface area element if $\Sigma=\partial \Xi$.

Proof. The first two properties (1.24) are obvious for the functional $\mathcal{F}: H^{1}(\Xi)^{k} \rightarrow$ $\mathbb{C}^{d}$ occurring on the right-hand side in (1.26) (cf. (1.23)), in particular, because the embedding $H^{1}(\Xi) \subset L_{2}(\Sigma)$ is compact. To verify the third property, we observe that the numerical $(d \times d)$-matrix

$$
\mathbf{P}(\Sigma)=\int_{\Sigma} \overline{\mathbf{p}(x)}^{\top} \mathbf{p}(x) d s_{x}
$$

is the Gram matrix constructed for the columns $\mathbf{p}^{1}, \ldots, \mathbf{p}^{d}$ of the matrix (1.15) with the help of the scalar product in $L_{2}(\Sigma)^{k}$. The matrix (1.27) is Hermitian, and it is positive definite provided the set $\Sigma$ has sufficiently many points to ensure that the polynomials $\mathbf{p}^{1}, \ldots, \mathbf{p}^{d}$ be linearly independent in $L_{2}(\Sigma)^{k}$.

Remark 1.2. The last phrase in the above proof demonstrates that inequality (1.26) remains true to a large extent for an arbitrary choice of a set $\Sigma \subset \bar{\Xi}$. In this way, putting $\Xi=\Omega(h)$ and $\Sigma=\Gamma(h)$ (see the definitions (1.3) and (1.5)), we can deduce inequality (1.22): the second term on the right in (1.26) is killed by the Dirichlet boundary condition (1.8).

The proof of inequality (1.22), as presented in Chapter 7 of the book [7, does not clarify the dependence of the factor $c(h)$ on the domain $\Omega(h)$ and, in particular, on the small parameter $h \in(0,1]$; the main result in $\S 2$ of the present paper will be as follows.

Theorem 1.1. The factor $c(h)$ in the Gärding-Korn inequality (1.22) satisfies the estimate

$$
c(h) \leq C,
$$

where $C$ depends on the sets $\varpi, \Omega$ and the matrix $\mathcal{D}$, but not on the size $h \in(0,1]$ of the perforation.

As a by-product, in Subsection 3 of $\S 2$ we check the next statement.

Proposition 1.1. The following trace inequality is valid:

$$
\left\|u ; L_{2}(\Upsilon(h))\right\|^{2} \leq c h^{-1}\left\|\mathcal{D}\left(\nabla_{x}\right) u ; L_{2}(\Omega(h))\right\|^{2}, \quad u \in \stackrel{\circ}{H}^{1}(\Omega(h) ; \Gamma(h))^{k},
$$

where $c$ is independent of $u$ and $h \leq 1$. 


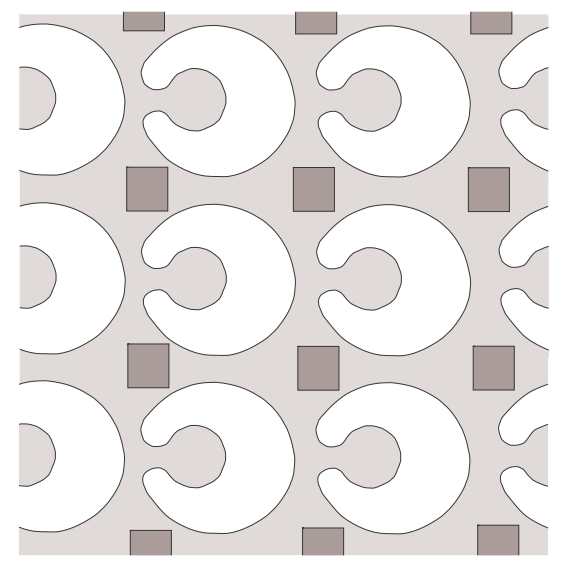

FiguRE 4

Theorem 1.1 is proved in Subsections 2 and 3 of $\S 2$. The proof employs the method developed in [9, 10] (see also the survey [11, Subsection 2.8]), more precisely, an extension of that method: what was considered in Example 1.2 were thin perforated plates (Example 1.2 and, in essence, the case of $n=2$ ). The basic idea is to form an auxiliary vector-valued function $v \in H^{1}\left(\mathbb{R}^{n}\right)^{k}$ with the following properties: $v$ is supported on the set

$$
\overline{\mathcal{S}(h)}=\{x: \operatorname{dist}(x, \partial \Omega) \leq h \sqrt{n}\} \cup \Omega ;
$$

on some periodic family of cubes $\left\{\mathbf{Q}_{h}(\alpha)\right\}_{\alpha \in \mathbb{Z}^{n}}$ in the perforated space (1.4) (the shaded parts in Figure 4; cf. Figure 2 and Figure 1, c)), $v$ coincides with the vector-valued function $u \in \stackrel{\circ}{H}^{1}(\Omega(h) ; \Gamma(h))^{k}$ extended by zero to $\Pi$, and $v$ admits the estimate

$$
\left\|\mathcal{D}\left(\nabla_{x}\right) v ; L_{2}\left(\mathbb{R}^{n}\right)\right\|^{2} \leq c\left\|\mathcal{D}\left(\nabla_{x}\right) u ; L_{2}(\Omega(h))\right\|^{2},
$$

with $c$ independent of $u$ and $h \leq 1$.

Lemma 1.3. If the support of a vector-valued function $v \in H^{1}\left(\mathbb{R}^{n}\right)^{k}$ lies in the compact set (1.30), then

$$
\left\|v ; H^{1}\left(\mathbb{R}^{n}\right)\right\|^{2} \leq c_{\mathcal{D}, \Omega}\left\|\mathcal{D}\left(\nabla_{x}\right) v ; L_{2}\left(\mathbb{R}^{n}\right)\right\|^{2},
$$

where $c_{\mathcal{D}, \Omega}$ is a constant.

Proof. Applying the Fourier transformation $v(x) \mapsto \widehat{v}(\xi)$ and the Parseval identity, we find

$$
\left\|\mathcal{D}\left(\nabla_{x}\right) v ; L_{2}\left(\mathbb{R}^{n}\right)\right\|^{2}=C \int_{\mathbb{R}^{n}}|\mathcal{D}(\xi) \widehat{v}(\xi)|^{2} d \xi
$$

We check that

$$
|\mathcal{D}(\xi) a|^{2} \geq c_{\mathcal{D}}|\xi|^{2}|a|^{2}, \quad a \in \mathbb{C}^{k}, \xi \in \mathbb{R}^{n}, c_{\mathcal{D}}>0
$$

then the desired inequality (1.32) can be deduced from relations (1.33), (1.34) and the Friedrichs inequality on a fixed ball containing the compact set (1.30) with $h=1$, after the inverse Fourier transformation.

Suppose (1.34) fails and there exist nonzero columns $a^{0} \in \mathbb{C}^{k}$ and $\xi^{0} \in \mathbb{R}^{n}$ such that $\mathcal{D}\left(\xi^{0}\right) a^{0}=0$. We put $p(\xi)=\left(a^{0}\right)^{\top}|\xi|^{2 \varrho \mathcal{D}}$ in identity (1.12) and multiply it scalarly from the right by the column $a^{0}$. For $\xi=\xi^{0}$ we have $\left|a^{0}\right|^{2}\left|\xi^{0}\right|^{2 \varrho_{\mathcal{D}}}=q\left(\xi^{0}\right) \mathcal{D}\left(\xi^{0}\right) a^{0}=0$, i.e., either $a^{0}=0$ or $\xi^{0}=0$. This contradiction finishes the proof of (1.34). 
Inequalities (1.31) and (1.32) imply that

$$
\left\|u ; H^{1}\left(\bigcup \mathbf{Q}_{h}(\alpha)\right)\right\|^{2} \leq c\left\|\mathcal{D}\left(\nabla_{x}\right) u ; L_{2}(\Omega(h))\right\|^{2} .
$$

Finally, an extension of estimate (1.35) from the union of cubes to the entire perforated domain $\Omega(h)$ is done with the help of a trick suggested in [12] (see also the survey [11, $\S 2])$ and modified here for the study of general systems with the polynomial property.

4. Asymptotics of the solution of the mixed boundary-value problem. In $\S 3$ we will give first terms of the formal asymptotic expansion of the solution of problem (1.6) -(1.8); this asymptotics is justified in $\S 4$.

Largely, the homogenization of the initial boundary-value problem in the perforated domain and the deduction of the limiting Dirichlet problem

$$
\begin{aligned}
\mathbf{L}\left(x, \nabla_{x}\right) \mathbf{u}(x) & =\mathbf{f}(x), & & x \in \Omega, \\
\mathbf{u}(x) & =0, & & x \in \partial \Omega,
\end{aligned}
$$

follow the standard lines: we construct the asymptotic corrector and work with solvability conditions for the model problem on the periodicity cell (see the monographs [13]- 16]). However, some distinctions arise, related not only to the passage to general formally selfadjoint systems, but also to the prescribed expansions of the right-hand sides:

$$
\begin{aligned}
f^{h}(x) & =h^{-1} F\left(x, h^{-1} x\right)+\left(\operatorname{meas}_{n} \varpi\right)^{-1} \mathbf{f}^{0}(x)+\widetilde{f}^{h}(x), \\
g^{h}(x) & =G\left(x, h^{-1} x\right)+\widetilde{g}^{h}(x),
\end{aligned}
$$

in which "large" components $F$ and $G$ are introduced, which are subject to the following orthogonality conditions:

$$
\int_{\varpi} F(x, y) d y+\int_{v} G(x, y) d s_{y}=0 \in \mathbb{C}^{k},
$$

for a.e. $x \in \Omega$, where $v=\partial \varpi \cap \mathbb{Q}$ is the inner surface of the cell, and $d s_{y}$ is the element of the $(n-1)$-dimensional surface area.

The asymptotic Ansatz, compatible with (1.38)-(1.40), for the solution of problem (1.6) - (1.8) looks like this:

$$
u^{h}(x)=\mathbf{u}(x)+h\left(\mathcal{X}\left(x, h^{-1} x\right) \mathcal{D}\left(\nabla_{x}\right) \mathbf{u}(x)+U\left(x, h^{-1} x\right)\right)+\widetilde{u}^{h}(x) .
$$

Here the corrector $\mathcal{X} \mathcal{D}\left(\nabla_{x}\right) \mathbf{u}$ involves a $(k \times N)$-matrix function $\mathcal{X}$, the columns of which are special periodic solutions of the model problem on the cell $\varpi$ (see Subsection 1 of $\S 3)$, but $U$ is the periodic solution of the same problem with the right-hand sides $F$ and $G$, which exists due to the orthogonality conditions (1.40) imposed above. The only (and rather simple) innovation in the Ansatz (1.41), namely, the term $U$, is caused by the presence of the terms $h^{-1} F$ and $G$ in the expansions (1.38) and (1.39). In elasticity theory (Example 1.2), such terms have a meaning of locked-up stresses, and the orthogonality condition (1.40), meaning that the load is self-balanced, arises naturally due to Newton's law.

Remark 1.3. Since our problem is linear, the imposed condition (1.40) is not burdensome. Indeed, in case it fails, put

$$
F(x, y)=F^{\perp}(x, y)+\left(\operatorname{meas}_{n} \varpi\right)^{-1} \mathbf{f}^{-1}(x), \mathbf{f}^{-1}(x)=\int_{\varpi} F(x, y) d y+\int_{v} G(x, y) d s_{y}
$$

and include the expressions $F^{\perp}\left(x, h^{-1} x\right)+\left(\operatorname{meas}_{n} \varpi\right)^{-1} \mathbf{f}^{0}(x)$ and $G\left(x, h^{-1} x\right)$ in the remainder terms, forming (respectively) the quantities $\widetilde{f}^{-1 h}(x)$ and $\widetilde{g}^{-1 h}(x)$ (cf. the end 
of Remark 1.4). Now formulas (1.38) and (1.39) look like this:

$$
\begin{aligned}
f^{h}(x)= & h^{-1}\left(\operatorname{meas}_{n} \varpi\right)^{-1} \mathbf{f}^{-1}(x) \\
& +h^{0}\left(h^{-1} F^{\perp}\left(x, h^{-1} x\right)+\left(\operatorname{meas}_{n} \varpi\right)^{-1} \mathbf{f}^{0}(x)\right)+\widetilde{f}^{h}(x) \\
= & h^{-1}\left(\operatorname{meas}_{n} \varpi\right)^{-1} \mathbf{f}^{-1}(x)+\widetilde{f}^{-1 h}(x), \\
g^{h}(x)= & G\left(x, h^{-1} x\right)+\widetilde{g}^{h}(x)=\widetilde{g}^{-1 h}(x) .
\end{aligned}
$$

Reading the new formulas without the middle terms, we can interpret them as the previous expansions (1.38), (1.39) multiplied by $h^{-1}$, with $F=0, G=0$, and $\mathbf{f}^{0}=\mathbf{f}^{-1}$. Since problem (1.6)-(1.8) is linear, the constant coefficient $h^{-1}$ is irrelevant. It should be noted that in the middle terms of (1.42) we easily find counterparts of structures occurring in (1.38), (1.39), because now the pair $\left\{F^{\perp}, G\right\}$ satisfies (1.40).

Throughout the entire paper, a number $\delta \in(0,1 / 2]$ will be fixed. The smallness condition for remainders is expressed by the formula

$$
\widetilde{\mathbf{n}}=h^{-\delta}\left(\left\|\widetilde{f}^{h} ; L_{2}(\Omega(h))\right\|+h^{-1 / 2}\left\|\widetilde{g}^{h} ; L_{2}(\Upsilon(h))\right\|\right),
$$

and we assume that $\widetilde{\mathbf{n}}$ is bounded as $h \rightarrow+0$. The factor $h^{-1 / 2}$ in the second term on the right in (1.43) is needed because the $L_{2}(\Omega(h))$-norm of the periodic function $\Omega(h) \ni$ $x \mapsto z\left(h^{-1} x\right)$ does not exceed $c\left\|z ; L_{2}(\varpi)\right\|$, but for the function $\Upsilon(h) \ni x \mapsto z\left(h^{-1} x\right)$ we only have the estimate

$$
\left\|z ; L_{2}(\Upsilon(h))\right\| \leq c h^{-1 / 2}\left\|z ; L_{2}(v)\right\| .
$$

The same factor arose in the trace inequality (1.29).

Remark 1.4. Of course, condition (1.43) can be relaxed. For instance, assuming that

$$
\widetilde{f}^{h}(x)=-\nabla_{x} \widetilde{f}^{h 1}(x)+\widetilde{f}^{h 0}(x), \quad \widetilde{g}^{h}(x)=\nu^{h}(x) \widetilde{f}^{h 1}(x)+\widetilde{g}^{h 0}(x),
$$

we can replace $h^{-\delta}\left\|\widetilde{f}{ }^{h 0} ; L_{2}(\Omega(h))\right\|$ in the definition of the quantity $\widetilde{\mathbf{n}}$ with the sum of $h^{-\delta}\left\|\tilde{f}^{h 1} ; L_{2}(\Omega(h))\right\|$ and $h^{-\delta}\left\|\widetilde{f}^{h} ; L_{2}(\Omega(h))\right\|$, and make the change

$$
\left(\widetilde{f}^{h}, v\right)_{\Omega(h)}+\left(\widetilde{g}^{h}, v\right)_{\partial \Omega(h)} \mapsto\left(\widetilde{f}^{h 1}, \nabla_{x} v\right)_{\Omega(h)}+\left(\widetilde{f}^{h 0}, v\right)_{\Omega(h)}+\left(\widetilde{g}^{h 0}, v\right)_{\partial \Omega(h)}
$$

in the integral identity (1.11). If we do not take care of differential properties of the right-hand sides, but do take care of their sizes, then the "remainder" can be taken to be "large"; i.e., by analogy with formulas (1.38)-(1.40), we can put

$$
\begin{gathered}
\widetilde{f}^{h}(x)=-h^{\delta-1} \widetilde{F}\left(x, h^{-1} x\right)+h^{\delta} \widetilde{f}^{0}(x), \quad \widetilde{g}^{h}(x)=h^{\delta} \widetilde{G}\left(x, h^{-1} x\right), \\
\int_{\varpi} \widetilde{F}(x, y) d y+\int_{v} \widetilde{G}(x, y) d s_{y}=0 \in \mathbb{C}^{k} .
\end{gathered}
$$

The vector-valued functions $F, G$, and $\mathbf{f}^{0}$ will be subject to restrictions of two types: higher smoothness requirements are imposed on the dependences on one group of variables, slow or fast. In both cases, such restrictions make all the distinguished terms of the sum (1.41) belong to the Sobolev space $H^{1}(\Omega(h))^{k}$, and for the intermediate asymptotic approximation, the boundary condition (1.8) is ensured by multiplication by a suitable cutoff function (see Subsection 1, §4). 
5. Statement of theorems on asymptotics. The Sobolev-Slobodetskiǔ and Hölder spaces of abstract functions with values in a Banach space $\mathfrak{B}$ are denoted by $H^{l+\alpha}(\Omega ; \mathfrak{B})$ and $C^{l, \alpha}(\Omega ; \mathfrak{B})$, where $l \in\{0,1,2, \ldots\}$ and $\alpha \in(0,1)$. The norms are as follows:

$$
\begin{aligned}
& \left\|z ; H^{l+\alpha}(\Omega ; \mathfrak{B})\right\| \\
& \quad=\left(\sum_{p=0}^{l} \int_{\Omega}\left\|\nabla_{x}^{p} z(x) ; \mathfrak{B}\right\|^{2} d x+\int_{\Omega} \int_{\Omega}\left\|\nabla_{x}^{l} z(x)-\nabla_{\mathbf{x}}^{l} z(\mathbf{x}) ; \mathfrak{B}\right\|^{2} \frac{d x d \mathbf{x}}{|x-\mathbf{x}|^{n+2 \alpha}}\right)^{1 / 2},
\end{aligned}
$$

$$
\begin{aligned}
& \left\|z ; C^{l, \alpha}(\Omega ; \mathfrak{B})\right\| \\
& \quad=\sum_{p=0}^{l} \sup _{x \in \Omega}\left\|\nabla_{x}^{p} z(x) ; \mathfrak{B}\right\|+\sup _{x, \mathbf{x} \in \Omega}\left(|x-\mathbf{x}|^{-\alpha}\left\|\nabla_{x}^{l} z(x)-\nabla_{\mathbf{x}}^{l} z(\mathbf{x}) ; \mathfrak{B}\right\|\right) .
\end{aligned}
$$

Here $\nabla_{x}^{p} z$ is the collection of all derivatives of order $p$ of a function $z$. If $\mathfrak{B}=\mathbb{C}$, then formulas (1.44) and (1.45) determine norms in the usual spaces $H^{l+\alpha}(\Omega)$ and $C^{l, \alpha}(\Omega)$. Also, we shall deal with the spaces $L_{\infty}(\Omega ; \mathfrak{B})$ and $H_{\infty}^{1}(\Omega ; \mathfrak{B})$; we put

$$
\begin{aligned}
\left\|z ; L_{\infty}(\Omega ; \mathfrak{B})\right\| & =\underset{x \in \Omega}{\operatorname{ess} \sup }\|z(x) ; \mathfrak{B}\|, \\
\left\|z ; H_{\infty}^{1}(\Omega ; \mathfrak{B})\right\| & =\left\|z ; L_{\infty}(\Omega ; \mathfrak{B})\right\|+\left\|\nabla_{x} z ; L_{\infty}(\Omega ; \mathfrak{B})\right\| .
\end{aligned}
$$

Clearly, $C^{0, \alpha}(\Omega ; \mathfrak{B}) \subset L_{\infty}(\Omega ; \mathfrak{B})$ and $C^{1, \alpha}(\Omega ; \mathfrak{B}) \subset H_{\infty}^{1}(\Omega ; \mathfrak{B})$ for any $\alpha>0$.

First of all, for the verification of asymptotic formulas we shall need the condition

$$
\partial \Omega \in C^{2, \delta_{\Omega}}, \quad \delta_{\Omega}>\delta .
$$

In the case of a higher smoothness relative to the fast variables, we assume that

$$
\begin{aligned}
& F \in H^{1+\delta}\left(\Omega ; C_{\mathrm{per}}^{0, \delta_{1}}(\varpi)\right)^{k}, G \in H^{1+\delta}\left(\Omega ; C_{\text {per }}^{1, \delta_{1}}(v)\right)^{k}, \mathbf{f}^{0} \in H^{\delta}(\Omega)^{k}, \delta_{1} \in(0,1), \\
& \mathcal{A} \in C^{1, \delta_{A}}\left(\Omega ; C_{\text {per }}^{1, \delta_{\varpi}}(\varpi)\right)^{N \times N}, \quad v \in C_{\text {per }}^{2, \delta_{\varpi}}, \quad \delta_{\varpi}>\delta_{A}>\delta_{1}, \quad \delta_{A}>\delta .
\end{aligned}
$$

Below, it will be checked that those conditions ensure that for the matrix $\mathbf{A}$ occurring in (1.36) we have

$$
\mathbf{A} \in C^{1, \delta_{A}}(\Omega)^{N \times N} .
$$

All the exponents $\delta_{1}, \delta_{\varpi}$ and $\delta_{A}, \delta_{\Omega}$ do not exceed 1 , and the first two can be taken arbitrarily small, but positive.

Theorem 1.2. Under conditions (1.46) -(1.48), the solution of problem (1.6)-(1.8) and its asymptotic approximation found by the solutions $\mathbf{u}$ and $U, \mathcal{X}$ of problems (1.36), (1.37) and (3.2), (3.3), respectively, are related as follows:

$$
\left\|u^{h}-\mathbf{u}-h \mathcal{X} \mathcal{D}\left(\nabla_{x}\right) \mathbf{u}-h U ; H^{1}(\Omega(h))\right\| \leq c_{H} h^{\delta}\left(\mathbf{n}_{H}+\widetilde{\mathbf{n}}\right),
$$

where $\mathbf{n}_{H}$ is the sum of the vector-valued functions (1.47) in the corresponding spaces, and $\widetilde{\mathbf{n}}$ is the quantity (1.43). The factor $c_{H}$ depends neither on the parameter $h \in\left(0, h_{0}\right]$, $h_{0}>0$, nor on the components $F, \mathbf{f}^{0}, \widetilde{f}^{h}$ and $G, \widetilde{g}^{h}$ in the expansions (1.38) and (1.39) of the right-hand sides of the problem in the perforated domain $\Omega(h)$.

Dealing with the differential properties relative to the slow variables, we impose the simplifying requirements

$$
\mathcal{A}(x, y)=\mathcal{A}(y), \quad F(x, y)=\mathbf{a}(x) F^{\prime}(y), \quad G(x, y)=\mathbf{a}(x) G^{\prime}(y) .
$$




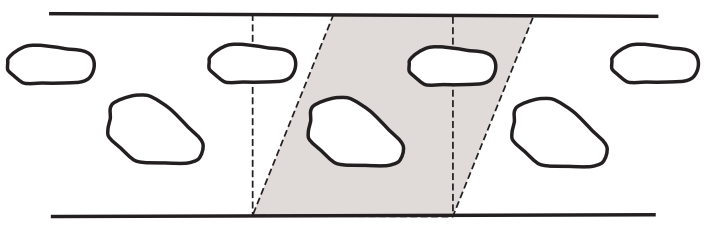

FIGURE 5

In other words, we assume that the coefficients of differential operators are periodic functions of the fast variables 2 and for the "large" terms $h^{-1} F$ and $G$ in (1.38) and (1.39), the dependences on the slow and fast variables are separated (a is a scalar). Also, we assume that

$$
\begin{aligned}
F^{\prime} \in L_{2}(\varpi)^{k}, \quad & G^{\prime} \in L_{2}(v)^{k}, \quad \mathbf{a} \in C^{1, \delta}(\Omega), \quad \mathbf{f}^{0} \in C^{0, \delta}(\Omega)^{k}, \\
\mathcal{A} & \in L_{\infty}(\varpi)^{N \times N}, \quad v \in \operatorname{Lip},
\end{aligned}
$$

which can be viewed as smoothness improvement relative to the slow variables, because the last two relations in (1.52) involve Hölder spaces in the domain $\Omega$.

Theorem 1.3. Under conditions (1.46), (1.51)-(1.53), the solution of problem (1.6)(1.8) and its asymptotic approximation are related as follows:

$$
\left\|u^{h}-\mathbf{u}-h \mathcal{X} \mathcal{D}\left(\nabla_{x}\right) \mathbf{u}-h U ; H^{1}(\Omega(h))\right\| \leq c_{C} h^{\delta}\left(\mathbf{n}_{C}+\widetilde{\mathbf{n}}\right),
$$

where $\widetilde{\mathbf{n}}$ is the quantity (1.43), and

$$
\mathbf{n}_{C}=\left\|\mathbf{f}^{0} ; C^{0, \delta}(\Omega)\right\|+\left\|\mathbf{a} ; C^{1, \delta}(\Omega)\right\|\left(\left\|F^{\prime} ; L_{2}(\varpi)\right\|+\left\|G^{\prime} ; L_{2}(v)\right\|\right) .
$$

The factor $c_{C}$ is independent of $h \in\left(0, h_{0}\right]$ and of $\mathbf{a}, F^{\prime}, \mathbf{f}^{0}, \widetilde{f}^{h}$, and $G^{\prime}, \widetilde{g}^{h}$.

Theorems 1.2 and 1.3 are proved in $\S 4$. Of course, in their proofs, the conditions imposed on the matrices $\mathcal{A}$ and $\mathcal{D}$ in Subsection 1 of $\S 1$ are assumed.

As usual, the asymptotic formulas can be simplified if the $L_{2}$-norms of the vectorvalued functions and their derivatives are treated separately.

Corollary 1.1. Under the assumptions of Theorems 1.2 and 1.3 , the norms

$$
\begin{aligned}
& \left\|u^{h}-\mathbf{u} ; L_{2}(\Omega(h))\right\|, \\
& \left\|\nabla_{x} u^{h}-\nabla_{x} \mathbf{u}-\left.\nabla_{y} \mathcal{X}\right|_{y=h^{-1} x} \mathcal{D}\left(\nabla_{x}\right) \mathbf{u}-\left.\nabla_{y} U\right|_{y=h^{-1} x} ; L_{2}(\Omega(h))\right\|
\end{aligned}
$$

admit the same majorants as in inequalities (1.50) and (1.54), respectively.

6. Comparison to known results. The homogenization of boundary-value problems in perforated domains was studied by many researchers (see the monographs [13, 14, 15, [16] and the references therein), who dealt mainly with scalar equations and elasticity theory problems (Examples 1.1 and 1.2). In all publications, the inner perforation condition was assumed, which means that the periodicity cell $\varpi$, possibly even not cubic or "skew" (see Remark 1.1), can be chosen so that its outer boundary $\partial \varpi \backslash v$ so that a solid $(n-1)$-dimensional surface, i.e., so that all holes lie strictly inside the cell (see Figure 1, and compare Figure 1, b) to Figure 5). In that case, the question about the PoincaréFriedrichs inequality in the scalar case and about the Korn inequality for the elasticity theory equations becomes elementary because an extension $v$ of the vector-valued function $u$, satisfying estimate (1.31), can be constructed by using local considerations only.

\footnotetext{
${ }^{2}$ We were forced to impose this restriction because of technical complications in the verification of the asymptotics (see [17). The authors do not know if Theorem 1.3 remains true under the condition of local periodicity for the coefficients.
} 


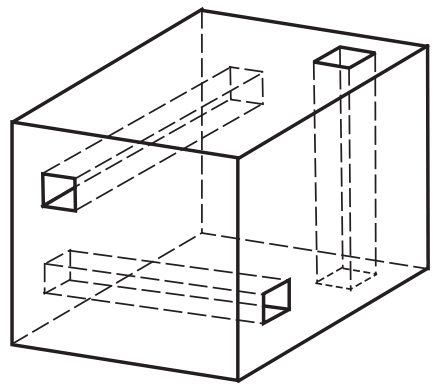

Figure 6

However, the inner perforation condition fails in many situations (see, e.g., Figure 6, which represents a cell in a 3-dimensional body pierced with a family of narrow channels parallel to the Cartesian axes). The results of the present paper allow us to get rid of the above-mentioned restriction, which is off the point.

Remark 1.5. Besides the mixed boundary-value problem (1.6)-(1.8), considered in [13[15] and in $\S 1$ of Chapter 2 in the book [16], in the same chapter of [16] we find the study of the Neumann problem for the system of equations of elasticity theory: the inner part of the boundary is assumed to be free of stresses, and loads are given on the outer part. Also, it is assumed that there is no perforation near the surface $\partial \Omega$ : the cells $\varpi_{h}(\alpha)$ not lying inside $\Omega$ are replaced with the sets $\mathbb{Q}_{h}(\alpha) \cap \Omega$, where $\mathbb{Q}_{h}(\alpha)=\left\{x: h^{-1} x-\alpha \in \mathbb{Q}\right\}$. The results of the present paper do not cover such a setting, because the Korn inequality with the constant (1.28) is proved here by a method that does not work in the case of an arbitrary perforation. Under the inner perforation condition, as mentioned above, inequality (1.28) for the Korn constant is obvious. Also, some bulk and hardly verifiable conditions can be presented (cf. [11, §2.4]) under which Theorem 1.1] remains true even if there is no boundary perforation.

Deflecting our attention away from the scalar problems, where additional smoothness is ensured by specific methods (see, e.g., [18]), we now discuss the smoothness conditions imposed in the book [16] on elasticity theory problems of that sort, set in an $n$-dimensional domain $\Omega(h)$ (i.e., $k=n$ and $N=n(n+1) / 2$ in Example 1.2). Namely, the matrix $\mathcal{A}$ of classic modules is assumed to be piecewise smooth, with smooth discontinuity surfaces not intersecting the smooth surface $v=\partial \varpi \cap \mathbb{Q}$. These requirements guarantee that the asymptotic corrector $\mathcal{X}$ is piecewise smooth, implying, in particular, that its derivatives are sufficiently bounded so as to ensure the second term in (1.41) to belong to the Sobolev space $H^{1}(\Omega(h))^{k}$. As to the right-hand sides, it is assumed that in (1.38) and (1.39) we have

$$
F=0, \quad G=0, \quad \mathbf{f}^{0} \in H^{1}(\Omega)^{n}, \quad \tilde{f}^{h} \in L_{2}(\Omega(h))^{n}, \quad \widetilde{g}^{h}=0 .
$$

Thus, $U=0$, which means that the locked-up stresses are absent, together with the elastic microfields. Finally, the smallness of the asymptotic remainders is expressed by inequality (1.50) with the majorant

$$
c\left(h^{1 / 2}\left\|\mathbf{f}^{0} ; H^{1}(\Omega)\right\|+\left\|\tilde{f}^{h} ; L_{2}(\Omega(h))\right\|\right) .
$$

The results of $\S 4$ allow us to lift the higher smoothness requirement imposed on the vector-valued function $\mathbf{f}^{0}$ (see (1.47) ): looking through the proofs in $\S 4$, we easily see that the requirement that the matrix $\mathcal{A}$ be piecewise smooth is quite sufficient for verifying Theorem 1.2 so that conditions (1.48) were imposed only for the simplification and unification of references. 


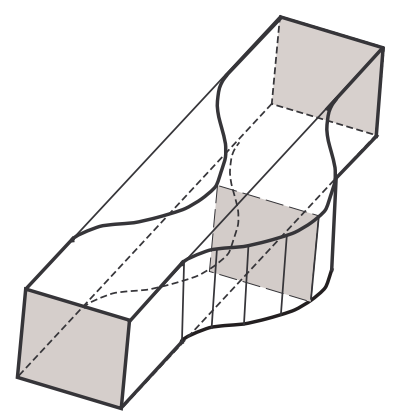

Figure 7

Apart from (1.47), (1.48), we pay attention to the restrictions (1.52), (1.53), which make only minimal demands on the dependences on the fast variables. In both cases, our conditions force the asymptotic corrector $x \mapsto \mathcal{X}\left(x, h^{-1} x\right) \mathcal{D}\left(\nabla_{x}\right) \mathbf{u}(x)$ to lie in the Sobolev space $H^{1}(\Omega(h))^{k}$, and precisely this fact predetermines essentially the conditions imposed on the data of the problem. It should be mentioned that the method developed in a series of publications [19-21] (see 22]-24] for an alternative approach) makes it possible, first, to obtain asymptotic expansions, together with estimates for the difference between the true solution and the approximate solution without invoking the corrector itself, and second, to construct an asymptotic corrector under minimal smoothness requirements. This method is based on applications of the spectral theory techniques for operators in Hilbert space. However, this method is adapted purely to differential equations with rapidly oscillating coefficients in the entire space $\mathbb{R}^{n}$ and compares the resolvents of the operators that correspond to the initial and the homogenized elliptic system. Therefore, the method of [19] 21] cannot be carried directly over to the case of the perforated space (1.4); it is an open question whether it applies to the Neumann problem for system (1.6) in $\Pi_{h}$. We note that homogenization problems in perforated domains were considered in [22, 24] for scalar equations and the elasticity theory systems with periodic coefficients under the same restriction on the cell as in the book [16].

\section{§2. The generalized Gåding-Korn inequalities and other estimates}

1. Geometric constructions for a perforated space. Let $\mathbf{Q}$ be a cube with small edge $l>0$ in the cell $\varpi$, and let $\mathbf{Q}(\alpha)$ be its integral shifts, $\alpha \in \mathbb{Z}^{n}$. The edges of $\mathbf{Q}=\mathbf{Q}(0)$ are assumed to be parallel to the coordinate axes. Since the set $\Pi$ is connected, the length $l$ can be fixed so small that we can transfer the cube from the position $\mathbf{Q}(0)$ to the position $\mathbf{Q}\left(e_{(j)}\right)$ so that its center moves along a smooth path and the cube itself remains within $\Pi$. As a result, we obtain sleeves $\mathbf{T}_{j}(0) \subset \Pi$ connecting the cube $\mathbf{Q}(0)$ to its neighbors $\mathbf{Q}\left(e_{(j)}\right), j=1, \ldots, n$ (recall that $e_{(j)}=\left(\delta_{j, 1}, \ldots, \delta_{j, n}\right)^{\top}$ is the unit vector of the $x_{j}$-axis). Copying the sleeves by periodicity, we obtain a family $\left\{\mathbf{T}_{j}(\alpha)\right\}_{\alpha \in \mathbb{Z}^{n}, j=1, \ldots, n}$.

Let $\mathbf{Q}(\alpha)$ and $\mathbf{Q}\left(\alpha+e_{(j)}\right)$ be two neighboring cubes; they are joined with the sleeve $\mathbf{T}_{j}(\alpha)$. Consider the brick $\mathbf{B}_{j}(\alpha)$ (Figure 7 ) obtained by the parallel translation of the cube along the segment that connects the centers of $\mathbf{Q}(\alpha)$ and $\mathbf{Q}\left(\alpha+e_{(j)}\right)$. Of course, part of the brick $\mathbf{B}_{j}(\alpha)$ may lie outside $\Pi$. Nevertheless, we have a continuous extension operator

$$
H^{1}\left(\mathbf{T}_{j}(\alpha)\right)^{k} \ni u \mapsto \gamma_{j} u \in H^{1}\left(\mathbf{T}_{j}(\alpha) \cup \mathbf{B}_{j}(\alpha)\right)^{k},
$$

because the boundary $\partial \mathbf{T}_{j}(\alpha)$ is Lipschitzian. Observe that, due to periodicity, we mark the operator $\gamma_{j}$ with only one index $j=1, \ldots, n$; for $j$ fixed, the extensions (2.1) are obtained by a unified rule for all $\alpha \in \mathbb{Z}^{n}$. Later, we shall need a certain specific extension. 
Suppose that a vector-valued function $u \in H^{1}(\Pi)^{k}$ has compact support. On the set $\mathbf{T}_{1}(\alpha) \cup \cdots \cup \mathbf{T}_{n}(\alpha)$, we represent this function in the form

$$
u(x)=u_{(\alpha)}^{\perp}(x)+\mathbf{p}\left(x-x_{(\alpha)}\right) u_{(\alpha)}^{0},
$$

where $\mathbf{p}$ is the matrix (1.15) corresponding to the basis $\mathbf{p}^{1}, \ldots, \mathbf{p}^{d}$ in $\mathcal{P}, u_{(\alpha)}^{0} \in \mathbb{C}^{d}$ is a column,

$$
\int_{\mathbf{Q}} \overline{\mathbf{p}(x)}^{\top} u_{(\alpha)}^{\perp}\left(x+x_{(\alpha)}\right) d x=0 \in \mathbb{C}^{d},
$$

and $x_{(\alpha)}$ is the center of the cube $\mathbf{Q}(\alpha)$. By the orthogonality conditions (2.3) and the versions (1.26) of the Korn inequality for $\Xi=\Sigma=\mathbf{Q}(\alpha)$ and for $\Xi=\mathbf{T}_{j}(\alpha), \Sigma=\mathbf{Q}(\alpha)$, applied to the vector-valued function $x \mapsto u_{(\alpha)}^{\perp}\left(x+x_{(\alpha)}\right)$, we have

$$
\begin{aligned}
\left\|u_{(\alpha)}^{\perp} ; H^{1}(\mathbf{Q}(\alpha))\right\|^{2} & \leq c\left\|\mathcal{D}\left(\nabla_{x}\right) u_{(\alpha)}^{\perp} ; L_{2}(\mathbf{Q}(\alpha))\right\|^{2}=c\left\|\mathcal{D}\left(\nabla_{x}\right) u ; L_{2}(\mathbf{Q}(\alpha))\right\|^{2}, \\
\left\|u_{(\alpha)}^{\perp} ; H^{1}\left(\mathbf{T}_{j}(\alpha)\right)\right\|^{2} & \leq c_{j}\left(\left\|\mathcal{D}\left(\nabla_{x}\right) u_{(\alpha)}^{\perp} ; L_{2}\left(\mathbf{T}_{j}(\alpha)\right)\right\|^{2}+\left\|u_{(\alpha)}^{\perp} ; L_{2}(\mathbf{Q}(\alpha))\right\|^{2}\right) \\
& \leq C_{j}\left\|\mathcal{D}\left(\nabla_{x}\right) u ; L_{2}\left(\mathbf{T}_{j}(\alpha)\right)\right\|^{2},
\end{aligned}
$$

where the component $u_{(\alpha)}^{\perp}$ of the expansion (2.2) is changed for the vector-valued function $u$ itself, which is possible by formula (1.14).

The desired extension $v^{1}$ is given by the identity

$$
v^{1}(x)=\mathbf{p}\left(x-x_{(\alpha)}\right) u_{(\alpha)}^{0}+\gamma_{j} u_{(\alpha)}^{\perp}(x), \quad x \in \mathbf{B}_{j}(\alpha),
$$

where $\gamma_{j}$ is the operator (2.1). The second estimate in (2.4) yields

$$
\begin{aligned}
& \left\|\mathcal{D}\left(\nabla_{x}\right) v^{1} ; L_{2}\left(\mathbf{B}_{j}(\alpha)\right)\right\|^{2}=\left\|\mathcal{D}\left(\nabla_{x}\right) \gamma_{j} u_{(\alpha)}^{\perp} ; L_{2}\left(\mathbf{B}_{j}(\alpha)\right)\right\|^{2} \\
& \quad \leq c_{j}\left\|u_{(\alpha)}^{\perp} ; H^{1}\left(\mathbf{T}_{j}(\alpha)\right)\right\|^{2} \leq C_{j}\left\|\mathcal{D}\left(\nabla_{x}\right) u ; L_{2}\left(\mathbf{T}_{j}(\alpha)\right)\right\|^{2} .
\end{aligned}
$$

Two important facts should be mentioned. First, $v^{1}$ is well defined on the union of all bricks

$$
\mathbf{B}=\bigcup_{\alpha \in \mathbb{Z}^{n}} \bigcup_{j=1}^{n} \mathbf{B}_{j}(\alpha)
$$

because $v^{1}=u$ on the common part of two intersecting bricks, which coincides with some cube $\mathbf{Q}(\beta)$ (see (2.2), (2.1) and (2.5)). Second, since the set (1.2) is periodic, we have $n$ different copies of the extension operator. Hence, summing estimates (2.6), taken without their middle parts, over $\alpha \in \mathbb{Z}^{n}$ and $j=1, \ldots, n$, we get

$$
\left\|\mathcal{D}\left(\nabla_{x}\right) v^{1} ; L_{2}(\mathbf{B})\right\|^{2} \leq c\left\|\mathcal{D}\left(\nabla_{x}\right) u ; L_{2}(\mathbf{T})\right\|^{2} \leq c_{1}\left\|\mathcal{D}\left(\nabla_{x}\right) u ; L_{2}(\Pi)\right\|^{2} ;
$$

here the union of sleeves $\mathbf{T} \subset \Pi$ is defined by formula (2.7) (with clear modifications). Observe that the resulting extension makes the convex hull of the support of the function larger, but the set

$$
\operatorname{supp} v^{1} \subset\{x: \operatorname{dist}(x, \operatorname{supp} u) \leq \sqrt{n}\}
$$

remains compact. The union (2.7) of bricks will be called a skeleton in $\mathbb{R}^{n}$ with support codimension 1 and will be denoted $\mathbf{K}^{1}$.

We fix a pair of indices $\theta=(j, k) \in\{1, \ldots, n\}^{2}$ and consider all cubes $\mathbf{Q}(\alpha)$ such that $\alpha_{j}, \alpha_{k} \in\{0,1\}$ and $\alpha_{p}=0$ for $p \neq j, k$. The bricks that join such cubes form a frame $\mathbf{F}_{\theta}^{1}(0) \subset \mathbf{K}^{1}$ (Figure 8) with directrix of codimension 1 (the support dimension of the frame). The convex hull $\mathbf{W}_{\theta}^{2}$ of the frame, i.e., a parallelepiped of size $1+l$ along the axes $x_{j}, x_{k}$ and of size $l$ in the other directions, is formed by the frame $\mathbf{F}_{\theta}^{1}(0)$ itself and the block $\mathbf{P}_{\theta}^{2}(0)$ bordered with that frame; $\mathbf{W}_{\theta}^{2}$ has support dimension 2 and volume $(1-l)^{2} l^{n-2}$. Replicating the solids introduced above by periodicity, we give meaning to 


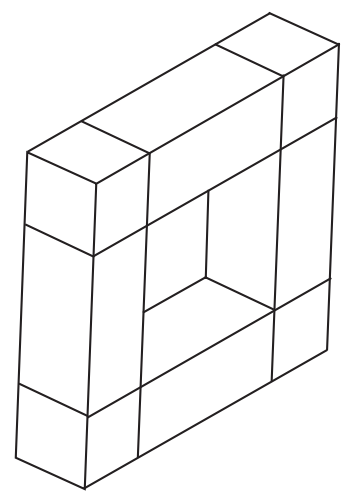

Figure 8

the objects $\mathbf{F}_{\theta}^{1}(\alpha), \mathbf{P}_{\theta}^{2}(\alpha)$, and $\mathbf{W}_{\theta}^{2}$ for $\alpha \in \mathbb{Z}^{2}, \theta \in\{1, \ldots, n\}^{2}$. The union of the sets $\mathbf{W}_{\theta}^{2}(\alpha)$ is the skeleton $\mathbf{K}^{2}$ with support dimension 2 .

Let $q=1$. Slightly modifying relations (2.2), (2.3), and (2.5), we construct an extension $v^{q+1}$ from the frames $\mathbf{F}_{\theta}^{q}(\alpha)$ with support dimension $q$ to the blocks $\mathbf{P}_{\theta}^{q+1}(\alpha)$ with support dimension $q+1$ by the formulas

$$
\begin{gathered}
v^{q}(x)=v_{(\theta \alpha)}^{q \perp}(x)+\mathbf{p}\left(x-x_{(\alpha)}\right) v_{(\theta \alpha)}^{q 0}, \quad x \in \mathbf{F}_{\theta}^{q}(\alpha), \\
\int_{\mathbf{Q}} \overline{\mathbf{p}(x)} v_{(\theta \alpha)}^{q \perp}\left(x+x_{(\alpha)}\right) d x=0 \in \mathbb{C}^{d}, \quad v_{(\theta \alpha)}^{q 0} \in \mathbb{C}^{N}, \\
v^{q+1}(x)=\gamma_{\theta}^{q} v_{(\theta \alpha)}^{q \perp}(x)+\mathbf{p}\left(x-x_{(\alpha)}\right) v_{(\theta \alpha)}^{q 0}, \quad x \in \mathbf{W}_{\theta}^{q+1}(\alpha),
\end{gathered}
$$

where $\gamma_{\theta}^{q}$ is the new extension operator

$$
H^{1}\left(\mathbf{F}_{j}^{q}(\alpha)\right)^{k} \ni u \mapsto \gamma_{\theta}^{q} u \in H^{1}\left(\mathbf{W}_{\theta}^{q+1}(\alpha)\right)^{k} .
$$

The same arguments as above show that the vector-valued function $v^{q+1} \in H^{1}\left(\mathbf{K}^{q+1}\right)^{k}$ is well defined on the skeleton

$$
\mathbf{K}^{q+1}=\bigcup_{\alpha \in \mathbb{Z}^{n}} \bigcup_{\theta \in\{1, \ldots, n\}^{q+1}} \mathbf{W}_{(\theta)}^{q+1}(\alpha),
$$

of support dimension $q+1$, and estimates similar to (2.4), (2.6) ensure the inequality

$$
\begin{aligned}
\left\|\mathcal{D}\left(\nabla_{x}\right) v^{q+1} ; L_{2}\left(\mathbf{K}^{q+1}\right)\right\|^{2} & \leq c_{q+1}\left\|\mathcal{D}\left(\nabla_{x}\right) v^{q} ; L_{2}\left(\mathbf{K}^{q}\right)\right\|^{2} \\
& \leq c_{q+1} c_{q} \ldots c_{1}\left\|\mathcal{D}\left(\nabla_{x}\right) u ; L_{2}(\Pi)\right\|^{2} .
\end{aligned}
$$

Observe also that the support of $v^{q+1}$ satisfies (2.8). The above formulas allow us to use induction ${ }^{3}$ to construct extensions $v^{2}, v^{3}, \ldots, v^{n}$ of the vector-valued function $v^{1}$ to the skeletons $\mathbf{K}^{2}, \mathbf{K}^{3}, \ldots, \mathbf{K}^{n}$ with growing support dimensions. Each $v^{p} \in H^{1}\left(\mathbf{K}^{p}\right)^{k}$ coincides with the initial vector-valued function $u$ on the cubes $\mathbf{Q}(\alpha), \alpha \in \mathbb{Z}^{n}$, and the last function $v=v^{n}$ satisfies (2.8) and the estimate

$$
\left\|\mathcal{D}\left(\nabla_{x}\right) v ; L_{2}\left(\mathbb{R}^{n}\right)\right\|^{2} \leq c\left\|\mathcal{D}\left(\nabla_{x}\right) u ; L_{2}(\Pi)\right\|^{2},
$$

which reshapes to (1.31) after the change $x \mapsto h^{-1} x$.

\footnotetext{
${ }^{3}$ The induction step was described in the last paragraph, and the induction base in the preceding paragraphs.
} 


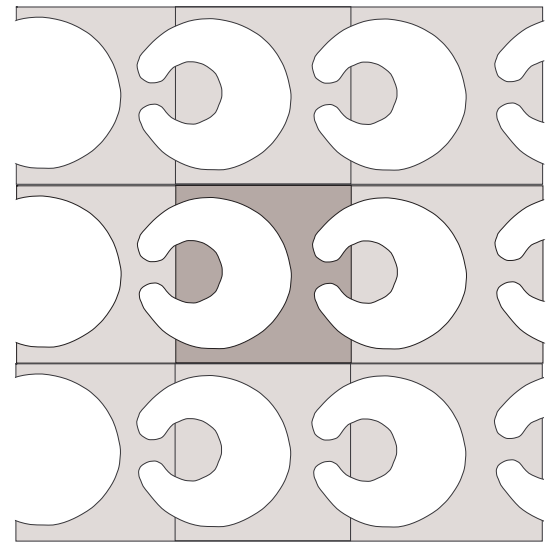

FiguRE 9

2. Proof of Theorem 1.1. Suppose a vector-valued function $u^{h} \in \stackrel{\circ}{H}^{1}(\Omega(h) ; \Gamma(h))^{k}$ is extended by zero to the perforated space (1.4). Put $u(y)=u^{h}(h y)$; clearly, we have

$$
\left\|\mathcal{D}\left(\nabla_{y}\right) u ; L_{2}(\Pi)\right\|^{2}=h^{2-n}\left\|\mathcal{D}\left(\nabla_{x}\right) u^{h} ; L_{2}\left(\Pi_{h}\right)\right\|^{2} .
$$

The procedure described in the preceding subsection allows us to form a vector-valued function $y \mapsto v(y)$ belonging to the Sobolev space $H^{1}\left(\mathbb{R}^{n}\right)^{k}$; we denote $v^{h}(x)=v\left(h^{-1} x\right)$. By (2.9), we have

$$
\begin{aligned}
\left\|\mathcal{D}\left(\nabla_{x}\right) v^{h} ; L_{2}\left(\mathbb{R}^{n}\right)\right\|^{2} & =h^{n-2}\left\|\mathcal{D}\left(\nabla_{y}\right) v ; L_{2}\left(\mathbb{R}^{n}\right)\right\|^{2} \\
& \leq c h^{n-2}\left\|\mathcal{D}\left(\nabla_{y}\right) u ; L_{2}(\Pi)\right\|^{2}=c\left\|\mathcal{D}\left(\nabla_{x}\right) u^{h} ; L_{2}(\Omega(h))\right\|^{2}
\end{aligned}
$$

(cf. (1.31)), and the support supp $v^{h}$ is contained in the set (1.30). Also, we have $u^{h}=v^{h}$ on the union $\mathbf{Q}_{h}$ of the small cubes $\mathbf{Q}_{h}(\alpha)$,

$$
\mathbf{Q}_{h}=\bigcup_{\alpha \in \mathbb{Z}^{n}} \mathbf{Q}_{h}(\alpha), \quad \mathbf{Q}_{h}(\alpha)=\left\{x: y=h^{-1} x \in \mathbf{Q}(\alpha)\right\} .
$$

Hence, by Lemma 1.3

$$
\begin{aligned}
& \left\|u^{h} ; L_{2}\left(\mathbf{Q}_{h}\right)\right\|^{2}+\left\|\nabla_{x} u^{h} ; L_{2}\left(\mathbf{Q}_{h}\right)\right\|^{2}=\left\|v^{h} ; H^{1}\left(\mathbf{Q}_{h}\right)\right\|^{2} \\
& \quad \leq c\left\|\mathcal{D}\left(\nabla_{x}\right) v^{h} ; L_{2}\left(\mathbb{R}^{n}\right)\right\|^{2} \leq c\left\|\mathcal{D}\left(\nabla_{x}\right) u^{h} ; L_{2}(\Omega(h))\right\|^{2} .
\end{aligned}
$$

It remains to extend estimate (2.11) from the union of the cubes (2.10) to the entire domain $\Omega(h)$.

We fix a cube $\mathbf{Q}_{h}(\alpha)$ and unite the closure of the corresponding compressed periodicity cell $\varpi_{h}(\alpha)$ and the closures of its $3^{n}-1$ immediate neighbors. Then, from the interior of the resulting set we draw the connected component $\varpi_{h}^{\bullet}(\alpha)$ that contains the cube $\mathbf{Q}_{h}(\alpha)$ (see Figure 9, where a disconnected, deeply shadowed periodicity cell is shown, the same as in Figure 1, c), and Figure 2, along with an extended cell $\varpi_{h}(\alpha) \subset \varpi_{h}^{\bullet^{*}}(\alpha)$ ).

Starting with the basis $\mathbf{p}^{1}, \ldots, \mathbf{p}^{d} \in \mathcal{P}$ constructed in Subsection 1 of $\S 1$ (see, in particular, (1.16)), we form a new basis:

$$
\begin{array}{ll}
\mathfrak{p}^{j}(h, x)=h^{-n / 2} e_{j} \in \mathbb{C}^{k}, & j=1, \ldots, k, \\
\mathfrak{p}^{j}(h, x)=h^{-n / 2}\left(\mathbf{p}^{j}\left(h^{-1} x\right)-\overline{\mathbf{p}}^{j}\right), & j=k+1, \ldots, d,
\end{array}
$$

where $\overline{\mathbf{p}}^{j}$ is the mean value of the polynomial $y \mapsto \mathbf{p}^{j}(y)$ over the cube $(-l / 2, l / 2)^{n}$ with edge $l$. We represent the vector-valued function $u^{h}$ on the extended cell $\varpi_{h}^{\bullet}(\alpha)$ in the 
form

$$
u^{h}(x)=u_{(\alpha)}^{h \perp}(x)+\mathfrak{p}\left(h, x-x_{(\alpha)}^{h}\right) u_{(\alpha)}^{h 0} .
$$

Here $x_{(\alpha)}^{h}$ is the center of the cube $\mathbf{Q}_{h}(\alpha), \mathfrak{p}=\left(\mathfrak{p}^{1}, \ldots, \mathfrak{p}^{d}\right)$ is a matrix of size $k \times d$,

$$
u_{(\alpha)}^{h 0}=\mathfrak{P}^{-1} \int_{\mathbf{Q}_{h}(\alpha)} \overline{\mathfrak{p}\left(h, x-x_{(\alpha)}^{h}\right)}{ }^{\top} u^{h}(x) d x,
$$

and $\mathfrak{P}$ is a Hermitian, positive definite $(d \times d)$-matrix,

$$
\mathfrak{P}=\int_{\mathbf{Q}_{h}(\alpha)}{\overline{\mathfrak{p}\left(h, x-x_{(\alpha)}^{h}\right)}}^{\top} \mathfrak{p}\left(h, x-x_{(\alpha)}^{h}\right) d x=\int_{(-l / 2, l / 2)^{n}} \overline{\mathfrak{p}(1, y)}^{\top} \mathfrak{p}(1, y) d y .
$$

Formulas (2.13) and (2.14) imply that

$$
\int_{\mathbf{Q}_{h}(\alpha)} \overline{\mathfrak{p}\left(h, x-x_{(\alpha)}^{h}\right)}{ }^{\top} u_{(\alpha)}^{h \perp}(x) d x=0 \in \mathbb{C}^{d} .
$$

We split the column $u_{(\alpha)}^{h 0} \in \mathbb{C}^{d}$ into two columns $a \in \mathbb{C}^{k}$ and $b \in \mathbb{C}^{d-k}$ in accordance with the two different formulas (2.12) for the elements of the basis $\mathfrak{p}^{1}, \ldots, \mathfrak{p}^{d}$. Observe that, first, $\mathfrak{p}^{1}, \ldots, \mathfrak{p}^{k}$ are constant columns, and second, $\mathfrak{P}=\operatorname{diag}\left\{\mathfrak{P}^{k}, \mathfrak{P}^{d-k}\right\}$ is a blockdiagonal matrix with blocks $\mathfrak{P}^{k}$ and $\mathfrak{P}^{d-k}$ of sizes $k \times k$ and $(d-k) \times(d-k)$, respectively. Now we estimate the components of the columns:

$$
\begin{aligned}
& \left|a_{j}\right|^{2} \leq c h^{-n}\left|\int_{\mathbf{Q}_{h}(\alpha)} u^{h}(x) d x\right|^{2} \leq c\left\|u^{h} ; L_{2}\left(\mathbf{Q}_{h}(\alpha)\right)\right\|^{2}, \quad j=1, \ldots, k, \\
& \left|b_{j}\right|^{2} \leq c h^{-n} \sum_{r=k+1}^{d}\left|\int_{\mathbf{Q}_{h}(\alpha)}{\overline{\left(\mathbf{p}^{r}\left(h^{-1}\left(x-x_{(\alpha)}^{h}\right)\right)-\overline{\mathbf{p}}^{r}\right)}}^{\top} u^{h}(x) d x\right|^{2} \\
& \leq c h^{-n} \sum_{r=k+1}^{d}\left|\int_{\mathbf{Q}_{h}(\alpha)}{\overline{\left(\mathbf{p}^{r}\left(h^{-1}\left(x-x_{(\alpha)}^{h}\right)\right)-\overline{\mathbf{p}}^{r}\right)}}^{\top}\left(u^{h}(x)-\bar{u}_{(\alpha)}^{h}\right) d x\right|^{2} \\
& \leq c\left\|u^{h}-\bar{u}_{(\alpha)}^{h} ; L_{2}\left(\mathbf{Q}_{h}(\alpha)\right)\right\|^{2} \leq c\left(\frac{l h}{\pi}\right)^{2}\left\|\nabla_{x}\left(u^{h}-\bar{u}_{(\alpha)}^{h}\right) ; L_{2}\left(\mathbf{Q}_{h}(\alpha)\right)\right\|^{2} \\
& =C h^{2}\left\|\nabla_{x} u^{h} ; L_{2}\left(\mathbf{Q}_{h}(\alpha)\right)\right\|^{2}, \quad j=k+1, \ldots, d .
\end{aligned}
$$

We have used the fact that, by the definition (2.12) of the vector polynomials $\mathfrak{p}^{k+1}, \ldots, \mathfrak{p}^{d}$, their mean values over the cube $(-h l / 2, h l / 2)^{n}$ are zero, so that the constant in the Poincaré inequality is $O\left(h^{2}\right)$. As a result, we obtain

$$
\begin{aligned}
\left\|\mathfrak{p} u_{(\alpha)}^{h 0} ; L_{2}\left(\varpi_{h}(\alpha)\right)\right\|^{2} & \leq c \operatorname{meas}_{n}\left(\varpi_{h}(\alpha)\right) h^{-n}\left(|a|^{2}+|b|^{2}\right) \\
& \leq c\left\|u^{h} ; H^{1}\left(\mathbf{Q}_{h}(\alpha)\right)\right\|^{2}, \\
\left\|\nabla_{x}\left(\mathfrak{p} u_{(\alpha)}^{h 0}\right) ; L_{2}\left(\varpi_{h}(\alpha)\right)\right\|^{2} & \leq c \sum_{r=k+1}^{d}\left|b_{p}\right|^{2} h^{-n} \int_{\mathbf{Q}_{h}(\alpha)}\left|\nabla_{x} \mathbf{p}^{r}\left(h^{-1}\left(x-x_{(\alpha)}^{h}\right)\right)\right|^{2} d x \\
& \leq c h^{-2}|b|^{2} \leq c\left\|\nabla_{x} u^{h} ; L_{2}\left(\mathbf{Q}_{h}(\alpha)\right)\right\|^{2} .
\end{aligned}
$$

To treat the component $u_{(\alpha)}^{h \perp}$ of the expansion (2.13), we make the change $x \mapsto y$ and apply Lemma 1.2 with $\Xi=\varpi^{\bullet}(\alpha)$ and $\Sigma=\mathbf{Q}(\alpha)$ (see Remark 1.2). After the inverse change $y \mapsto x$, we see that inequality (1.26), in which the last term is absent due to the orthogonality condition (2.15), turns into the estimate

$$
\begin{aligned}
& h^{-2}\left\|u_{(\alpha)}^{h \perp} ; L_{2}\left(\varpi_{h}^{\bullet}(\alpha)\right)\right\|^{2}+\left\|\nabla_{x} u_{(\alpha)}^{h \perp} ; L_{2}\left(\varpi_{h}^{\bullet}(\alpha)\right)\right\|^{2} \\
& \quad \leq c\left\|\mathcal{D}\left(\nabla_{x}\right) u_{(\alpha)}^{h \perp} ; L_{2}\left(\varpi_{h}^{\bullet}(\alpha)\right)\right\|^{2}=c\left\|\mathcal{D}\left(\nabla_{x}\right) u^{h} ; L_{2}\left(\varpi_{h}^{\bullet}(\alpha)\right)\right\|^{2} .
\end{aligned}
$$


Observe that on the left-hand side of (2.18) we have $h^{-2} \geq 1$, because $h \leq 1$.

Collecting estimates (2.13), (2.17), and (2.18) and recalling that $\varpi_{h}(\alpha) \subset \varpi_{h}^{\bullet}(\alpha)$, we deduce the inequalities

$$
\begin{aligned}
\left\|u^{h} ; L_{2}\left(\varpi_{h}(\alpha)\right)\right\|^{2} & +\left\|\nabla_{x} u^{h} ; L_{2}\left(\varpi_{h}(\alpha)\right)\right\|^{2} \\
& \leq c\left(\left\|\mathcal{D}\left(\nabla_{x}\right) u^{h} ; L_{2}\left(\varpi_{h}^{\bullet}(\alpha)\right)\right\|^{2}+\left\|u^{h} ; H^{1}\left(\mathbf{Q}_{h}(\alpha)\right)\right\|^{2}\right) .
\end{aligned}
$$

Now we sum these estimates over all periodicity cells that intersect the perforated domain $\Omega(h)$, obtaining

$$
\left\|u^{h} ; H^{1}(\Omega(h))\right\|^{2} \leq c\left(3^{n}\left\|\mathcal{D}\left(\nabla_{x}\right) u^{h} ; L_{2}(\Omega(h))\right\|^{2}+\left\|u^{h} ; H^{1}\left(\mathbf{Q}_{h}\right)\right\|^{2}\right) .
$$

The factor of $3^{n}$ arose because of the enlarged cell $\varpi_{h}^{\bullet}(\alpha)$. Relations (2.19) and (2.11) ensure inequality (1.22) with a constant independent of the parameter $h \in(0,1]$. This proves estimate (1.28) and Theorem 1.1.

3. Proof of Proposition 1.1. The trace inequality (1.29) is checked along the same lines, but with simplifications: in particular, the proof involves only the constant columns in the basis (2.12) of the linear space $\mathcal{P}$. On the enlarged cell $\varpi_{h}^{\bullet}(\alpha)$ we use the expansion

$$
u^{h}(x)=w_{(\alpha)}^{h \perp}(x)+w_{(\alpha)}^{h 0},
$$

where $w_{(\alpha)}^{h 0}$ is the mean value of $u^{h}$ over the cube $\mathbf{Q}_{h}(\alpha) \subset \varpi_{h}^{\bullet}(\alpha)$, which admits the estimate

$$
\left|w_{(\alpha)}^{h 0}\right|^{2} \leq\left(\operatorname{meas}_{n} \mathbf{Q}_{h}\right)^{-1}\left\|u^{h} ; L_{2}\left(\mathbf{Q}_{h}(\alpha)\right)\right\|^{2} .
$$

When considering the component $w_{(\alpha)}^{h \perp}$ of the expansion (2.20), which has zero mean over the cube $\mathbf{Q}_{h}(\alpha)$, we apply the Poincaré and Friedrichs inequalities for the dilated coordinates $y=h^{-1} x$ :

$$
\begin{aligned}
& \left\|w_{(\alpha)}^{h \perp} ; L_{2}\left(\mathbf{Q}_{h}(\alpha)\right)\right\|^{2} \leq c h^{2}\left\|\nabla_{x} w_{(\alpha)}^{h \perp} ; L_{2}\left(\mathbf{Q}_{h}(\alpha)\right)\right\|^{2}=c h^{2}\left\|\nabla_{x} u^{h} ; L_{2}\left(\mathbf{Q}_{h}(\alpha)\right)\right\|^{2}, \\
& \begin{aligned}
h^{-2}\left\|w_{(\alpha)}^{h \perp} ; L_{2}\left(\varpi_{h}(\alpha)\right)\right\|^{2} & \leq h^{-2}\left\|w_{(\alpha)}^{h \perp} ; L_{2}\left(\varpi_{h}^{\bullet}(\alpha)\right)\right\|^{2} \\
& \leq c\left(\left\|\nabla_{x} w_{(\alpha)}^{h \perp} ; L_{2}\left(\varpi_{h}^{\bullet}(\alpha)\right)\right\|^{2}+h^{-2}\left\|w_{(\alpha)}^{h \perp} ; L_{2}\left(\mathbf{Q}_{h}(\alpha)\right)\right\|^{2}\right) .
\end{aligned}
\end{aligned}
$$

Combining these estimates with the usual trace inequality (see, e.g., the textbook [1]), again written in the dilated coordinates,

$$
h^{-1}\left\|w_{(\alpha)}^{h \perp} ; L_{2}\left(v_{h}(\alpha)\right)\right\|^{2} \leq c\left(\left\|\nabla_{x} w_{(\alpha)}^{h \perp} ; L_{2}\left(\varpi_{h}(\alpha)\right)\right\|^{2}+h^{-2}\left\|w_{(\alpha)}^{h \perp} ; L_{2}\left(\varpi_{h}(\alpha)\right)\right\|^{2}\right),
$$

and also with formulas (2.21) and (2.20), we obtain

$$
\begin{aligned}
h\left\|u^{h} ; L_{2}\left(v_{h}(\alpha)\right)\right\|^{2} & \leq 2 h\left(\operatorname{meas}_{n-1}\left(v_{h}\right)\left|w_{(\alpha)}^{h 0}\right|^{2}+\left\|w_{(\alpha)}^{h \perp} ; L_{2}\left(v_{h}(\alpha)\right)\right\|^{2}\right) \\
& \leq c\left(\left\|u^{h} ; L_{2}\left(\mathbf{Q}_{h}(\alpha)\right)\right\|^{2}+\left\|\nabla_{x} u^{h} ; L_{2}\left(\varpi_{h}^{\bullet}(\alpha)\right)\right\|^{2}\right) .
\end{aligned}
$$

Now, to arrive at the desired formula (1.29) and, thereby, finish the proof of Proposition 1.1, it suffices to sum the above inequalities (without their middle parts) over all cells that intersect the domain $\Omega(h)$ and use the already established estimates (2.19) and (2.11).

4. Weighted inequalities. The following simple estimates are based on versions of the one-dimensional Hardy inequality; the proofs can be found, e.g., in 6, Proposition 1.2.3 and Lemma 1.2.4]. 
Lemma 2.1. Suppose that the surface $\partial \Omega$ is Lipschitzian, $v \in \stackrel{\circ}{H}^{1}(\Omega ; \partial \Omega)$, and $w \in$ $H^{1}(\Omega)$. Let $\theta_{h}=\{x \in \Omega$ : dist $(x, \partial \Omega)<c h\}$ and $\Theta(h)=\Omega(h) \cap \theta_{h}$ be narrow boundary strips in the domains $\Omega$ and $\Omega(h)$, solid and perforated, respectively. Then

$$
\begin{aligned}
&\left\|v ; L_{2}(\Theta(h))\right\| \leq\left\|v ; L_{2}\left(\theta_{h}\right)\right\| \leq c h\left\|v ; H^{1}(\Omega)\right\|, \\
&\left\|w ; L_{2}(\Theta(h))\right\| \leq\left\|w ; L_{2}\left(\theta_{h}\right)\right\| \leq c h^{1 / 2}\left\|w ; H^{1}(\Omega)\right\|,
\end{aligned}
$$

where the constants $c$ depend neither on the functions involved, nor on the parameter $h \in\left(0, h_{0}\right]$.

The estimate

$$
\left\|u^{h} ; L_{2}(\Theta(h))\right\| \leq c h\left\|u^{h} ; H^{1}(\Omega(h))\right\|,
$$

similar to (2.23), is valid for any vector-valued function $u^{h} \in \stackrel{\circ}{H}^{1}(\Omega(h) ; \Gamma(h))^{k}$, due to another weighted inequality (the weight factor exceeds $c h^{-1}>0$ on the narrow strip $\Theta(h)$ ).

Lemma 2.2. We have

$$
\left\|\rho_{h}^{-1} u^{h} ; L_{2}(\Theta(h))\right\| \leq c\left\|\mathcal{D}\left(\nabla_{x}\right) u^{h} ; L_{2}(\Omega(h))\right\|, \quad u^{h} \in \stackrel{\circ}{H}^{1}(\Omega(h) ; \Gamma(h))^{k},
$$

where $\rho_{h}(x)=h+\operatorname{dist}(x, \partial \Omega)$, and $c$ is independent of $h \leq 1$ and $u^{h}$.

Proof. We mention the simple consequence

$$
\begin{array}{r}
\left\|(\operatorname{dist}(\cdot, \partial \mathcal{S}(h)))^{-1} v^{h} ; L_{2}(\mathcal{S}(h))\right\| \leq c\left\|\mathcal{D}\left(\nabla_{x}\right) v^{h} ; L_{2}(\mathcal{S}(h))\right\|, \\
\\
v^{h} \in \stackrel{\circ}{H}^{1}(\mathcal{S}(h) ; \partial \mathcal{S}(h))^{k},
\end{array}
$$

of the one-dimensional Hardy inequality

$$
\int_{0}^{\infty} t^{-2}|V(t)|^{2} d t \leq 4 \int_{0}^{\infty}\left|\frac{d V}{d t}(t)\right|^{2} d t, \quad V \in C_{c}^{\infty}(0, \infty) .
$$

Here $\overline{\mathcal{S}(h)}$ is the set (1.30); clearly, we have

$$
\operatorname{dist}(x, \partial \mathcal{S}(h)) \leq \sqrt{n} \rho_{h}(x) .
$$

Now, if in Subsection 2 of $\S 2$ we apply inequality (2.27) in place of the Gårding inequality (1.32), then, instead of (2.11), we obtain

$$
\left\|\rho_{h}^{-1} u^{h} ; L_{2}\left(\mathbf{Q}_{h}\right)\right\|^{2}+\left\|\nabla_{x} u^{h} ; L_{2}\left(\mathbf{Q}_{h}\right)\right\|^{2} \leq c\left\|\mathcal{D}\left(\nabla_{x}\right) u^{h} ; L_{2}(\Omega(h))\right\|^{2} .
$$

When extending estimate (2.28) from the union (2.10) of cubes to the entire perforated domain $\Omega(h)$, we turn to the expansion (2.13) on the cell $\varpi_{h}(\alpha)$, handling its components with the help of (2.16) and (2.18):

$$
\begin{aligned}
\left\|\rho_{h}^{-1} u^{h} ; L_{2}\left(\varpi_{h}(\alpha)\right)\right\|^{2} \leq & 2\left(\left\|\rho_{h}^{-1} u_{(\alpha)}^{h \perp} ; L_{2}\left(\varpi_{h}(\alpha)\right)\right\|^{2}+\left\|\rho_{h}^{-1} \mathfrak{p} u_{(\alpha)}^{h 0} ; L_{2}\left(\varpi_{h}(\alpha)\right)\right\|^{2}\right) \\
\leq & c\left(h+\operatorname{dist}\left(x_{(\alpha)}^{h}, \partial \Omega\right)\right)^{-2}\left(\left\|u_{(\alpha)}^{h \perp} ; L_{2}\left(\varpi_{h}(\alpha)\right)\right\|^{2}+\left|u_{(\alpha)}^{h 0}\right|^{2}\right) \\
\leq & c\left(h+\operatorname{dist}\left(x_{(\alpha)}^{h}, \partial \Omega\right)\right)^{-2}\left(h^{2}\left\|\mathcal{D}\left(\nabla_{x}\right) u^{h} ; L_{2}\left(\varpi_{h}^{\bullet}(\alpha)\right)\right\|^{2}\right. \\
& \left.\quad+h^{2}\left\|\nabla_{x} u^{h} ; L_{2}\left(\mathbf{Q}_{h}(\alpha)\right)\right\|^{2}+\left\|u^{h} ; L_{2}\left(\mathbf{Q}_{h}(\alpha)\right)\right\|^{2}\right) \\
\leq & c\left(\left\|\mathcal{D}\left(\nabla_{x}\right) u^{h} ; L_{2}\left(\varpi_{h}^{\bullet}(\alpha)\right)\right\|^{2}+\left\|\nabla_{x} u^{h} ; L_{2}\left(\mathbf{Q}_{h}(\alpha)\right)\right\|^{2}\right. \\
& \left.+\left\|\rho_{h}^{-1} u^{h} ; L_{2}\left(\mathbf{Q}_{h}(\alpha)\right)\right\|^{2}\right) .
\end{aligned}
$$

Besides the obvious inequality $h+\operatorname{dist}\left(x_{(\alpha)}^{h}, \partial \Omega\right) \geq h$, we have used the fact that for $x \in \mathbf{Q}_{h}(\alpha)$ the quantity $\rho_{h}(x)$ has the same order as the quantity $\rho_{h}\left(x_{(\alpha)}^{h}\right)$ calculated at the center $x_{(\alpha)}^{h}$ of the cube $\mathbf{Q}_{h}(\alpha)$. It remains to sum estimates (2.29) (without their middle parts) over all cells. 
The next inequality serves as a substitute of estimate (2.24) in the case of a function given on a perforated domain.

Lemma 2.3. If $z \in H^{1}\left(\Omega ; L_{\infty}(\varpi)\right)$, then

$$
\left\|z ; L_{2}(\Theta(h))\right\| \leq c h^{1 / 2}\left\|z ; H^{1}\left(\Omega ; L_{\infty}(\varpi)\right)\right\| .
$$

Proof. We extend the function $x \mapsto z(x, y)$ by zero beyond the domain $\Omega$ and denote by $\sum_{\alpha}$ the summation over all cells $\varpi_{h}(\alpha)$ that intersect $\Theta(h)$. We have

$$
\begin{aligned}
& \left\|z ; L_{2}(\Theta(h))\right\|^{2} \leq \sum_{\alpha} \int_{\varpi_{h}(\alpha)}\left|z\left(x, \frac{x}{h}\right)\right|^{2} d x \\
& \quad \leq \sum_{\alpha} \int_{\varpi_{h}(\alpha)} \sup _{y \in \varpi}|z(x, y)|^{2} d x \leq \int_{\{x \in \Omega: \operatorname{dist}(x, \partial \Omega) \leq \sqrt{n} c h\}} \sup _{y \in \varpi}|z(x, y)|^{2} d x \\
& \quad \leq \operatorname{ch} \int_{\Omega}\left(\sup _{y \in \varpi}|z(x, y)|^{2}+\sup _{y \in \varpi}\left|\nabla_{x} z(x, y)\right|^{2}\right) d x \leq c h\left\|z ; H^{1}\left(\Omega ; ; L_{\infty}(\varpi)\right)\right\|^{2} .
\end{aligned}
$$

We have applied the second statement of Lemma 2.1 .

Next, we employ the method used in the proof of Lemma 2.3 to deduce an inequality for the trace of a vector-valued function $u$ on the part $\Upsilon(h) \cap \partial \Theta(h)$ of the surface of the thin set $\Theta(h)$.

Lemma 2.4. We have

$$
\left\|u ; L_{2}(\Upsilon(h) \cap \partial \Theta(h))\right\| \leq c h^{1 / 2}\left\|\mathcal{D}\left(\nabla_{x}\right) u ; L_{2}(\Omega(h))\right\|,
$$

where $c$ is independent of $u \in \stackrel{\circ}{H}^{1}(\Omega(h) ; \Gamma(h))^{k}$ and $h \in(0,1]$.

Proof. As usual, we extend $u$ by zero from $\Omega(h)$ to $\Pi_{h}$; let $\sum_{\alpha}$ mean the same as in (2.30). Observe that the coordinate compression $y \mapsto h y$ in the standard trace inequality on a cell of size 1 (see, e.g., [1]) results in the relation

$$
h^{-1}\left\|u ; L_{2}\left(v_{h}(\alpha)\right)\right\|^{2} \leq c\left(h^{-2}\left\|u ; L_{2}\left(\varpi_{h}(\alpha)\right)\right\|^{2}+\left\|\mathcal{D}\left(\nabla_{x}\right) u ; L_{2}\left(\varpi_{h}(\alpha)\right)\right\|^{2}\right) .
$$

Summing over all cells that intersect $\Theta(h)$ and using (2.26), we get

$$
\begin{aligned}
h^{-1}\left\|u ; L_{2}(\Upsilon(h) \cap \partial \Theta(h))\right\|^{2} & \leq c h^{-1} \sum_{\alpha}\left\|u ; L_{2}\left(v_{h}(\alpha)\right)\right\|^{2} \\
& \leq c \sum_{\alpha}\left(h^{-2}\left\|u ; L_{2}\left(\varpi_{h}(\alpha)\right)\right\|^{2}+\left\|\mathcal{D}\left(\nabla_{x}\right) u ; L_{2}\left(\varpi_{h}(\alpha)\right)\right\|^{2}\right) \\
& \leq c\left(\left\|\rho_{h}^{-1} u ; L_{2}(\Theta(\sqrt{n} h))\right\|^{2}+\left\|\mathcal{D}\left(\nabla_{x}\right) u ; L_{2}(\Theta(\sqrt{n} h))\right\|^{2}\right) \\
& \left.\leq c\left\|\mathcal{D}\left(\nabla_{x}\right) u ; L_{2}(\Omega(h))\right\|^{2}\right) .
\end{aligned}
$$

\section{§3. Formal Asymptotics And Differential PROPERTIES OF SOLUTions OF LIMITING PROBLEMS}

1. First asymptotic correction. As usual, when doing formal asymptotic analysis, we assume that all problem data are infinitely smooth.

We plug (1.41), (1.38), and (1.39) into (1.6), (1.7), and use the chain rule

$$
\mathcal{D}\left(\nabla_{x}\right) w\left(x, h^{-1} x\right)=\left.\left(h^{-1} \mathcal{D}\left(\nabla_{y}\right) w(x, y)+\mathcal{D}\left(\nabla_{x}\right) w(x, y)\right)\right|_{y=h^{-1} x} .
$$

Collecting the coefficients of $h^{-1}$ in differential equations and the coefficients of $h^{0}$ in the boundary conditions, we arrive at two model problems on the periodicity cell $\varpi$, 
depending on the parameter $x \in \Omega$, namely, to the problem

$$
\begin{aligned}
L^{0}\left(x, y, \nabla_{y}\right) U(x, y) & :={\overline{\mathcal{D}\left(-\nabla_{y}\right)}}^{\top} \mathcal{A}(x, y) \mathcal{D}\left(\nabla_{y}\right) U(x, y)=F(x, y), \quad y \in \varpi, \\
N^{0}\left(x, y, \nabla_{y}\right) U(x, y) & :=\overline{\mathcal{D}(\nu(y))}^{\top} \mathcal{A}(x, y) \mathcal{D}\left(\nabla_{y}\right) U(x, y)=G(x, y), \quad y \in v,
\end{aligned}
$$

for a vector-valued function $U$, and to a problem for the asymptotic corrector,

$$
\begin{aligned}
& L^{0}\left(x, y, \nabla_{y}\right) \mathcal{X}(x, y)=-{\overline{\mathcal{D}\left(-\nabla_{y}\right)}}^{\top} \mathcal{A}(x, y), \quad y \in \varpi, \\
& N^{0}\left(x, y, \nabla_{y}\right) \mathcal{X}(x, y):=-\overline{\mathcal{D}(\nu(y))}^{\top} \mathcal{A}(x, y), \quad y \in v .
\end{aligned}
$$

Here $\nu(y)$ is the unit vector (column) of the outward normal to the part $v=\partial \varpi \cap \mathbb{Q}$ of the cell surface. It should be noted that, in relations (3.3) and (1.6), the coefficients mentioned above and giving rise to identities (1.7) were multiplied from the right by the columns $\mathcal{D}\left(\nabla_{x}\right) \mathbf{u}(x)$. Since formulas (3.3) only involve differential operators in the variables $y=\left(y_{1}, \ldots, y_{n}\right)^{\top}$, and the variables $x=\left(x_{1}, \ldots, x_{n}\right)^{\top}$ serve as a parameter, the latter columns should be viewed as constants. Also, we note that the solution and the right-hand sides of problem (3.3) are matrix-valued functions of size $k \times N$.

Both problems (3.2) and (3.3) should be supplemented by periodicity conditions, which we do not write down explicitly. The variational version of problem (3.2),

$$
\left(\mathcal{A D}\left(\nabla_{y}\right) U, \mathcal{D}\left(\nabla_{y}\right) V\right)_{\varpi}=(F, V)_{\varpi}+(G, V)_{v}, \quad V \in H_{\text {per }}^{1}(\varpi)^{k},
$$

is posed on the subspace $H_{\mathrm{per}}^{1}(\varpi)$ of functions belonging to the Sobolev class and 1periodic relative to the variables $y_{j}, j=1, \ldots, n$. For clear reasons (see, e.g., Proposition 3.2 below), identity (3.2) can serve as a solvability condition for problem (1.40). As to problem (3.3), a similar solvability condition is fulfilled, due to the periodicity of the function $y \mapsto \mathcal{A}(x, y)$ and the Gauss-Ostrogradskiu formula

$$
\int_{\varpi}{\overline{\mathcal{D}\left(\nabla_{y}\right)}}^{\top} \mathcal{A}(x, y) d y=\int_{v} \overline{\mathcal{D}(\nu(y))}^{\top} \mathcal{A}(x, y) d s_{y} .
$$

Since the solutions of (3.2) and (3.3) are determined up to additive constants, the orthogonality conditions can be ensured:

$$
\begin{aligned}
& \int_{\varpi} U(x, y) d y=0 \in \mathbb{C}^{k}, \\
& \int_{v} \mathcal{X}(x, y) d y=0 \in \mathbb{C}^{k \times N} .
\end{aligned}
$$

Thus, we have found both correction terms in the Ansatz (1.41).

2. Higher order asymptotic correction. We supplement the asymptotic Ansatz for the solution of problem (1.6)-(1.8) with the term $h^{2} w\left(x, h^{-1} x\right)$ (actually, it will not be used in asymptotic approximation) and perform the same procedure as in the previous subsection, collecting the coefficients of $h^{0}$ in the differential equations (1.6) and the coefficients of $h^{1}$ in the Neumann boundary conditions (1.7), and using the expansions (1.38), (1.39) with negligibly small remainders $\widetilde{f}^{h}, \widetilde{g}^{h}$. As a result, we obtain the following model problem:

$$
\begin{aligned}
L^{0}\left(x, y, \nabla_{y}\right) w(x, y)= & -L^{1}\left(x, y, \nabla_{x}, \nabla_{y}\right) \mathcal{X}(x, y) \mathcal{D}\left(\nabla_{x}\right) \mathbf{u}(x) \\
& -L^{2}\left(x, y, \nabla_{x}\right) \mathbf{u}(x)+\mathcal{F}(x, y), \quad y \in \varpi, \\
N^{0}\left(x, y, \nabla_{y}\right) w(x, y)= & -N^{1}\left(x, y, \nabla_{x}\right) \mathcal{X}(x, y) \mathcal{D}\left(\nabla_{x}\right) \mathbf{u}(x)+\mathcal{G}(x, y), \quad y \in v .
\end{aligned}
$$


Here we have denoted

$$
\begin{aligned}
L^{1}\left(x, y, \nabla_{x}, \nabla_{y}\right) & ={\overline{\mathcal{D}\left(-\nabla_{y}\right)}}^{\top} \mathcal{A}(x, y) \mathcal{D}\left(\nabla_{x}\right)+{\overline{\mathcal{D}\left(-\nabla_{x}\right)}}^{\top} \mathcal{A}(x, y) \mathcal{D}\left(\nabla_{y}\right), \\
L^{2}\left(x, y, \nabla_{x}\right) & ={\overline{\mathcal{D}\left(-\nabla_{x}\right)}}^{\top} \mathcal{A}(x, y) \mathcal{D}\left(\nabla_{x}\right), \\
N^{1}\left(x, y, \nabla_{x}\right) & =\overline{\mathcal{D}(\nu(y))}^{\top} \mathcal{A}(x, y) \mathcal{D}\left(\nabla_{x}\right),
\end{aligned}
$$

and also

$$
\begin{aligned}
\mathcal{F}(x, y) & =\left(\operatorname{meas}_{n} \varpi\right)^{-1} \mathbf{f}^{0}(x)-L^{1}\left(x, y, \nabla_{x}, \nabla_{y}\right) U(x, y), \\
\mathcal{G}(x, y) & =-N^{1}\left(x, y, \nabla_{x}\right) U(x, y) .
\end{aligned}
$$

We find the solvability conditions for problem (3.8). We have

$$
\begin{aligned}
& \int_{\varpi} L^{1}\left(x, y, \nabla_{x}, \nabla_{y}\right) \mathcal{X}(x, y) \mathcal{D}\left(\nabla_{x}\right) \mathbf{u}(x) d y \\
& \quad+\int_{v} N^{1}\left(x, y, \nabla_{x}\right) \mathcal{X}(x, y) \mathcal{D}\left(\nabla_{x}\right) \mathbf{u}(x) d s_{y}+\int_{\varpi} L^{2}\left(x, y, \nabla_{x}\right) \mathbf{u}(x) d y \\
&={\overline{\mathcal{D}}\left(-\nabla_{x}\right)}^{\top} \int_{\varpi}\left(\mathcal{A}(x, y)+\mathcal{A}(x, y) \mathcal{D}\left(\nabla_{y}\right) \mathcal{X}(x, y)\right) d y \mathcal{D}\left(\nabla_{x}\right) \mathbf{u}(x) .
\end{aligned}
$$

Also, using (3.9) and (3.10), we obtain

$$
\begin{aligned}
\int_{\varpi} \mathcal{F}(x, y) d y+\int_{v} \mathcal{G}(x, y) d s_{y} \\
=\mathbf{f}^{0}(x)+{\overline{\mathcal{D}\left(\nabla_{x}\right)}}^{\top} \int_{\varpi} \mathcal{A}(x, y) \mathcal{D}\left(\nabla_{y}\right) U(x, y) d y \\
\quad+\int_{\varpi}{\overline{\mathcal{D}\left(\nabla_{y}\right)}}^{\top} \mathcal{A}(x, y) \mathcal{D}\left(\nabla_{x}\right) U(x, y) d y \\
\quad-\int_{v} \overline{\mathcal{D}(\nu(y))}
\end{aligned}
$$

The last two integrals mutually cancel, by a formula similar to (3.5), because the integrands are periodic. Continuing transformations, we find that the quantity (3.12) is equal to

$$
\begin{aligned}
& \mathbf{f}^{0}(x)+{\overline{\mathcal{D}\left(\nabla_{x}\right)}}^{\top}\left(-\int_{\varpi} U(x, y)^{\top} \mathcal{D}\left(\nabla_{y}\right)^{\top} \mathcal{A}(x, y) d y\right. \\
& \left.+\int_{v} U(x, y)^{\top} \mathcal{D}(\nu(y))^{\top} \mathcal{A}(x, y) d s_{y}\right)^{\top} \\
& =\mathbf{f}^{0}(x)-{\overline{\mathcal{D}\left(\nabla_{x}\right)}}^{\top}\left(\int_{\varpi} U(x, y)^{\top} \overline{L^{0}\left(x, y, \nabla_{y}\right) \mathcal{X}(x, y)} d y\right. \\
& \left.+\int_{v} U(x, y)^{\top} \overline{N^{0}\left(x, y, \nabla_{y}\right) \mathcal{X}(x, y)} d s_{y}\right)^{\top} \\
& =\mathbf{f}^{0}(x)-{\overline{\mathcal{D}\left(\nabla_{x}\right)}}^{\top}\left(\int_{\varpi} \overline{\mathcal{X}(x, y)}^{\top} F(x, y) d y+\int_{v} \overline{\mathcal{X}(x, y)}^{\top} G(x, y) d s_{y}\right) \\
& =: \mathbf{f}^{0}(x)-{\overline{\mathcal{D}\left(\nabla_{x}\right)}}^{\top} \mathbf{F}(x)=: \mathbf{f}(x),
\end{aligned}
$$

where

$$
\mathbf{F}(x)=\int_{\varpi} \overline{\mathcal{X}(x, y)}^{\top} F(x, y) d y+\int_{v} \overline{\mathcal{X}(x, y)}^{\top} G(x, y) d s_{y} .
$$


So, the solvability conditions for (3.8) take the form of the system of differential equations (1.36) in which the right-hand side is given by (3.13), and

$$
\begin{aligned}
\mathbf{L}\left(x, \nabla_{x}\right) & ={\overline{\mathcal{D}\left(-\nabla_{x}\right)}}^{\top} \mathbf{A}(x) \mathcal{D}\left(\nabla_{x}\right), \\
\mathbf{A}(x) & =\int_{\varpi} \mathcal{A}(x, y) d y+\int_{\varpi} \mathcal{A}(x, y) \mathcal{D}\left(\nabla_{y}\right) \mathcal{X}(x, y) d y .
\end{aligned}
$$

If the vector-valued function $\mathbf{u}$ satisfies (1.36), then problem (3.8) becomes solvable, but its solution $w$ will not be needed, because to justify the asymptotics (see $\S 4$ ) it suffices to check that the mean value over the periodicity cell of one of the discrepancy terms is zero. Precisely this fact is ensured by system (1.36).

3. Homogenized problem. The boundary conditions (1.37) correspond to the Dirichlet conditions in the initial problem. We turn to properties of the matrix (3.15).

Lemma 3.1. The matrix $\mathbf{A}(x)$ is Hermitian and positive definite.

Proof. We use the periodicity of the matrix-valued functions $y \mapsto \mathcal{A}(x, y), \mathcal{X}(x, y)$ and relations (3.15) to reshape the right-hand side of (3.3) as follows:

$$
\begin{aligned}
& \mathbf{A}=\int_{\varpi} \mathcal{A} d y+\int_{\varpi} \mathcal{A D} \mathcal{X} d y+\int_{\varpi} \overline{\mathcal{D X}}^{\top} \mathcal{A} d y-\int_{\varpi} \overline{\mathcal{D}}^{\top} \mathcal{A} d y \\
& =\int_{\varpi} \mathcal{A} d y+\int_{\varpi} \mathcal{A D} \mathcal{X} d y+\int_{\varpi} \overline{\mathcal{D}} \mathcal{X}^{\top} \mathcal{A} d y+\int_{\varpi} \overline{\mathcal{X}}^{\top} \overline{\mathcal{D}}^{\top} \mathcal{A} d y-\int_{v} \overline{\mathcal{X}}^{\top} \overline{\mathcal{D}}(\nu)^{\top} \mathcal{A} d s_{y} \\
& =\int_{\varpi} \mathcal{A} d y+\int_{\varpi} \mathcal{A D} \mathcal{X} d y+\int_{\varpi} \overline{\mathcal{D X}}^{\top} \mathcal{A} d y-\int_{\varpi} \overline{\mathcal{X}}^{\top} \overline{\mathcal{D}}^{\top} \mathcal{A} \mathcal{D} \mathcal{X} d y+\int_{v} \overline{\mathcal{X}}^{\top} \overline{\mathcal{D}}(\nu)^{\top} \mathcal{A} \mathcal{D} \mathcal{X} d s_{y} \\
& =\int_{\varpi} \mathcal{A} d y+\int_{\varpi} \mathcal{A D} \mathcal{X} d y+\int_{\varpi} \overline{\mathcal{D X}}^{\top} \mathcal{A} d y+\int_{\varpi} \overline{\mathcal{D X}}^{\top} \mathcal{A D} \mathcal{X} d y .
\end{aligned}
$$

As a result,

$$
\mathbf{A}(x)=\int_{\varpi}{\overline{\left(\mathbb{I}_{N}+\mathcal{D}\left(\nabla_{y}\right) \mathcal{X}(x, y)\right)}}^{\top} \mathcal{A}(x, y)\left(\mathbb{I}_{N}+\mathcal{D}\left(\nabla_{y}\right) \mathcal{X}(x, y)\right) d y,
$$

where $\mathbb{I}_{N}$ is the unit matrix of size $N \times N$.

Formula (3.16) shows that $\mathbf{A}(x)$ is Hermitian and nonnegative definite. If this matrix is singular, then, since the matrix $\mathcal{A}$ is positive definite, we have

$$
a+\mathcal{D}\left(\nabla_{y}\right) \mathcal{X}(x, y) a=0, \quad y \in \varpi
$$

where $a \in \mathbb{C}^{N}$ is a nontrivial numerical column. Recalling property (1.12) of the matrix $\mathcal{D}$, we see that

$$
0=q\left(\nabla_{y}\right)\left(a+\mathcal{D}\left(\nabla_{y}\right) \mathcal{X}(x, y) a\right)=q\left(\nabla_{y}\right) \mathcal{D}\left(\nabla_{y}\right) \mathcal{X}(x, y) a=p\left(\nabla_{y}\right) \mathcal{X}(x, y) a
$$

for any row $p=\left(p_{1}, \ldots, p_{k}\right)$ of homogeneous differential operators of degree $\max \left\{\varrho_{\mathcal{D}}, 2\right\}$. Observe that $q\left(\nabla_{x}\right)=0$, because $\operatorname{deg} q=\operatorname{deg} p-1 \geq 1$. Finally, we see that $y \mapsto$ $\mathcal{X}(x, y) a$ is a vector-valued polynomial of degree $\varrho_{\mathcal{D}}-1$, and hence, a constant column, by periodicity. Now formula (3.17) implies that $a=0$. The contradiction obtained shows that the matrix (3.16) is positive definite.

4. Solvability of the homogenized problem. The variational setting of the Dirichlet problem (1.36), (1.37) addresses the integral identity [1]

$$
\left(\mathbf{A} \mathcal{D}\left(\nabla_{x}\right) \mathbf{u}, \mathcal{D}\left(\nabla_{x}\right) \mathbf{v}\right)_{\Omega}=\left(\mathbf{f}^{0}, \mathbf{v}\right)_{\Omega}+\left(\mathbf{F}, \mathcal{D}\left(\nabla_{x}\right) \mathbf{v}\right)_{\Omega}, \quad \mathbf{v} \in \stackrel{\circ}{H}^{1}(\Omega ; \partial \Omega)^{k}
$$


Since the Gårding inequality (1.32) is valid without any smoothness restrictions on the surface $\partial \Omega$ (see the proof of Lemma 1.3), the generalized solution $\mathbf{u} \in \stackrel{\circ}{H}^{1}(\Omega ; \partial \Omega)^{k}$ exists and is unique for any

$$
\mathbf{f}^{0} \in L_{2}(\Omega)^{k}, \quad \mathbf{F} \in L_{2}(\Omega)^{N} .
$$

As to the matrix $\mathbf{A}$, it suffices that its entries be measurable and bounded, and the matrix itself be Hermitian and uniformly positive definite for a.e. $x \in \Omega$ (see Lemma 3.1). The solution $\mathbf{u} \in \stackrel{\circ}{H}^{1}(\Omega ; \partial \Omega)^{k}$ admits the estimate

$$
\left\|\mathbf{u} ; H^{1}(\Omega)\right\| \leq c \mathbf{n}_{0},
$$

where $\mathbf{n}_{0}$ is the sum of the $L_{2}$-norms of the vector-valued functions (3.19).

In the next section, for justifying the asymptotics we shall need to impose some additional smoothness requirements on the solution $\mathbf{u}$. We formulate the corresponding statements, implied by the general results of the theory of elliptic boundary-value problems (see, e.g., 25, 26]). We emphasize that, by Lemma 3.1, the sesquilinear Hermitian form on the left in (3.18) still has the polynomial property (1.13), and the $(k \times k)$-matrix of second order differential operators remains to be elliptic (see [2, 3, 4,).

Proposition 3.1. Under conditions (1.46) and (1.49), suppose that $\mathbf{f} \in H^{\delta}(\Omega)^{k}(\mathbf{f} \in$ $\left.C^{0, \delta}(\Omega)^{k}\right)$. Then the solution $\mathbf{u} \in \stackrel{\circ}{H}^{1}(\Omega ; \partial \Omega)^{k}$ of problem (1.36), (1.37) belongs to the Sobolev-Slobodetskiu space $H^{2+\delta}(\Omega)^{k}$ (to the Hölder space $\left.C^{2, \delta}(\Omega)^{k}\right)$, and its norm in that space does not exceed $c\left\|\mathbf{f} ; H^{\delta}(\Omega)\right\|\left(c\left\|\mathbf{f} ; C^{0, \delta}(\Omega)\right\|\right)$, with constant $c$ independent of the right-hand side $\mathbf{f}$ of system (1.36).

5. Solvability of the problem on the periodicity cell. Concise proofs of the following well-known facts are presented here for the reader's convenience.

Proposition 3.2. 1) Under conditions (1.53), suppose that the vector-valued functions $F \in L_{2}(\varpi)^{k}, G \in L_{2}(v)^{k}$ satisfy (1.40). Then the variational problem (3.4) has a solution $U \in H_{\mathrm{per}}^{1}(\varpi)^{k}$, determined uniquely up to a constant summand belonging to $\mathbb{C}^{k}$. If this solution is subject to the orthogonality conditions (3.6), then it is unique and satisfies

$$
\left\|U ; H^{1}(\varpi)\right\|^{2} \leq c\left(\left\|F ; L_{2}(\varpi)\right\|^{2}+\left\|G ; L_{2}(v)\right\|^{2}\right) .
$$

2) Under conditions (1.47), (1.48), and (1.40), the weak solution $U \in H_{\mathrm{per}}^{1}(\varpi)^{k}$ of problem (3.2) that exists for a.e. $x \in \Omega$ and satisfies the orthogonality condition (3.6) falls into the space $H^{1+\delta}\left(\Omega ; C_{\text {per }}^{2, \delta_{1}}(\varpi)\right)^{k}$, and

$$
\begin{aligned}
\left\|U ; H^{1+\delta}\left(\Omega ; C_{\text {per }}^{2, \delta_{1}}(\varpi)\right)\right\| & \\
& \leq c\left(\left\|F ; H^{1+\delta}\left(\Omega ; C_{\text {per }}^{0, \delta_{1}}(\varpi)\right)\right\|+\left\|G ; H^{1+\delta}\left(\Omega ; C_{\text {per }}^{1, \delta_{1}}(v)\right)\right\|\right) .
\end{aligned}
$$

Proof. Consider the auxiliary problem

$$
\left(\mathcal{A D}\left(\nabla_{y}\right) U, \mathcal{D}\left(\nabla_{y}\right) V\right)_{\varpi}+\mu(U, V)_{\varpi}=(F, V)_{\varpi}+(G, V)_{v}, \quad V \in H_{\mathrm{per}}^{1}(\varpi)^{k},
$$

assuming that $\mu \geq 0$. By inequality (1.21), for (3.22) for $\mu>0$ the left-hand side of $(3.22)$ is an inner product in the space $H_{\text {per }}^{1}(\varpi)^{k}$; hence, by the Riesz theorem on the representation of a functional on a Hilbert space, problem (3.22) is uniquely solvable for $\mu>0$. Since the embedding $H_{\text {per }}^{1}(\varpi) \subset L_{2}(\varpi)$ is compact, for $\mu=0$ the Fredholm alternative is valid for problem $(3.22)=(3.4)$. Any solution $U$ of the homogeneous $(F=0$, $G=0$ ) problem (3.4) nullifies the form $a(U, U ; \varpi)$, i.e., in accordance with property (1.13), falls into the linear space $\mathcal{P}$ of polynomials and turns out to be a constant column, by periodicity. The solvability conditions (1.40) coincide with the requirement that the 
right-hand side be orthogonal to the solutions of the homogeneous problem, and the restriction (3.6) removes the arbitrariness in the choice of a solution.

2) By the general results of [25, 26, the conditions (1.48) imposed on $\mathcal{A}$ and $v$ imply that the solution $U \in H_{\text {per }}^{1}(\varpi)^{k}$ of the integral identity (3.4) with $\left.F \in C_{\text {per }}^{0, \delta_{1}}(\varpi)\right)^{k}$ and $G \in C_{\text {per }}^{1, \delta_{1}}(v)^{k}$ belongs to $C_{\text {per }}^{2, \delta_{1}}(\varpi)^{k}$, satisfies (3.2), and is subject to the estimate

$$
\left\|U ; C_{\mathrm{per}}^{2, \delta_{1}}(\varpi)\right\|^{2} \leq c\left(\left\|F ; C_{\mathrm{per}}^{0, \delta_{1}}(\varpi)\right\|^{2}+\left\|G ; C_{\mathrm{per}}^{1, \delta_{1}}(v)\right\|^{2}+\left\|U ; L_{2}(\varpi)\right\|^{2}\right) .
$$

Observe that the last term on the right can be removed from (3.6), by the orthogonality condition (3.20) and inequality (3.23). Also, by (1.47) and (1.48), the solution $U(x, y)$ exists for a.e. $x \in \Omega$, and, what is important, the constants $c$ in (3.23) (and in (3.20) can be chosen to be common for all $x \in \Omega$. Thus, after integration over the variables $x \in \Omega$, we obtain

$$
\left\|U ; L_{2}\left(\Omega ; C_{\text {per }}^{2, \delta_{1}}(\varpi)\right)\right\|^{2} \leq c\left(\left\|F ; L_{2}\left(\Omega ; C_{\text {per }}^{0, \delta_{1}}(\varpi)\right)\right\|^{2}+\left\|G ; L_{2}\left(\Omega ; C_{\text {per }}^{1, \delta_{1}}(v)\right)\right\|^{2}\right) .
$$

Put $U^{(j)}=\partial U / \partial x_{j}$, and so on. Note that

$$
\mathcal{A}^{j} \in C^{0, \delta_{A}}\left(\Omega ; C_{\text {per }}^{1, \delta_{\varpi}}(\varpi)\right)^{N \times N}
$$

The problem

$$
\begin{aligned}
& L^{0}\left(x, y, \nabla_{y}\right) U^{(j)}(x, y)=\mathcal{F}^{j}(x, y) \\
& \quad:=\overline{\mathcal{D}\left(\nabla_{y}\right)}{ }^{\top} \mathcal{A}^{(j)}(x, y) \mathcal{D}\left(\nabla_{y}\right) U(x, y)+F^{(j)}(x, y), \quad y \in \varpi, \\
& N^{0}\left(x, y, \nabla_{y}\right) U^{(j)}(x, y)=\mathcal{G}^{j}(x, y) \\
& \quad:=-\overline{\mathcal{D}(\nu(y))}^{\top} \mathcal{A}^{(j)}(x, y) \mathcal{D}\left(\nabla_{y}\right) U(x, y)+G^{(j)}(x, y), \quad y \in v,
\end{aligned}
$$

obtained by formal differentiation with respect to $x_{j}$, has a unique periodic solution $U^{(j)} \in C_{\text {per }}^{2, \delta_{1}}(\varpi)^{k}$ for a.e. $x \in \Omega$, because, by conditions (1.47), (1.48) and estimate (3.24), we have

$$
\begin{aligned}
& \left.\left\|\mathcal{F}^{j} ; L_{2}\left(\Omega ; C_{\text {per }}^{0, \delta_{1}}(\varpi)\right)\right\|+\left\|\mathcal{G}^{j} ; L_{2}\left(\Omega ; C_{\text {per }}^{1, \delta_{1}}(v)\right)\right\|\right) \\
& \quad \leq c\left(\left\|U ; L_{2}\left(\Omega ; C_{\text {per }}^{2, \delta_{1}}(\varpi)\right)\right\|+\left\|F ; H^{1}\left(\Omega ; C_{\text {per }}^{0, \delta_{1}}(\varpi)\right)\right\|+\left\|G ; H^{1}\left(\Omega ; C_{\text {per }}^{1, \delta_{1}}(v)\right)\right\|\right),
\end{aligned}
$$

and the norm $\left\|U^{(j)} ; H^{1}\left(\Omega ; C_{\text {per }}^{2, \delta_{1}}(\varpi)\right)\right\|$ is also dominated by the right-hand side of (3.26) . Note that differentiation in $x_{j}$ leaves the orthogonality conditions (1.40) and (3.6) untouched; hence, the solution $U^{(j)}$ of problem (3.25) is the derivative $\partial U / \partial x_{j}$, and we have

$$
\left\|U ; H^{1}\left(\Omega ; C_{\text {per }}^{2, \delta_{1}}(\varpi)\right)\right\|^{2} \leq c\left(\left\|F ; H^{1}\left(\Omega ; C_{\text {per }}^{0, \delta_{1}}(\varpi)\right)\right\|^{2}+\left\|G ; H^{1}\left(\Omega ; C_{\text {per }}^{1, \delta_{1}}(v)\right)\right\|^{2}\right)
$$

For $x, \mathbf{x} \in \Omega$, consider the differences

$$
\triangle U^{(j)}(x, \mathbf{x}, y)=|x-\mathbf{x}|^{-\delta}\left(U^{(j)}(x, y)-U^{(j)}(\mathbf{x}, y)\right)
$$

\footnotetext{
${ }^{4}$ It would be more traditional to consider the difference $\triangle_{\ell} U(x, y)=|\ell|^{-1}(U(x+\ell, y)-U(x, y))$, to let $|\ell| \rightarrow 0, \ell \in \mathbb{R}^{n}$, in the estimate implied by (3.24), and to integrate over $x \in \Omega$ (see the subsequent arguments).
} 
and so on. We start with equations (3.25), subtract the same equations written at the point $\mathbf{x}$, and divide the result by $|x-\mathbf{x}|^{-\delta}$, arriving at the problem

$$
\begin{aligned}
& L^{0}\left(x, y, \nabla_{y}\right) \triangle U^{(j)}(x, \mathbf{x}, y)={\overline{\mathcal{D}\left(\nabla_{y}\right)}}^{\top} \triangle \mathcal{A}^{(j)}(x, \mathbf{x}, y) \mathcal{D}\left(\nabla_{y}\right) U(x, y) \\
& \quad+{\overline{\mathcal{D}\left(\nabla_{y}\right)}}^{\top} \triangle \mathcal{A}(x, \mathbf{x}, y) \mathcal{D}\left(\nabla_{y}\right) U^{(j)}(\mathbf{x}, y) \\
& \quad+{\overline{\mathcal{D}\left(\nabla_{y}\right)}}^{\top} \mathcal{A}^{(j)}(\mathbf{x}, y) \mathcal{D}\left(\nabla_{y}\right) \triangle U(x, \mathbf{x}, y)+\triangle F^{(j)}(x, \mathbf{x}, y), \quad y \in \varpi, \\
& N^{0}\left(x, y, \nabla_{y}\right) \triangle U^{(j)}(x, \mathbf{x}, y)=-\overline{\mathcal{D}}(\nu(y))^{\top} \triangle \mathcal{A}^{(j)}(x, \mathbf{x}, y) \mathcal{D}\left(\nabla_{y}\right) U(x, y) \\
& \quad-\overline{\mathcal{D}(\nu(y))}^{\top} \triangle \mathcal{A}(x, \mathbf{x}, y) \mathcal{D}\left(\nabla_{y}\right) U^{(j)}(\mathbf{x}, y) \\
& \quad-\overline{\mathcal{D}(\nu(y))}^{\top} \mathcal{A}^{(j)}(\mathbf{x}, y) \mathcal{D}\left(\nabla_{y}\right) \triangle U(x, \mathbf{x}, y)+\triangle G^{(j)}(x, \mathbf{x}, y), \quad y \in v .
\end{aligned}
$$

Now we apply inequality (3.24) and also, when treating the right-hand sides of problem (3.28), the already established inequalities (3.24) and (3.27). Integrating the result over the variables $x, \mathrm{x} \in \Omega$ and recalling the definition (1.44), we deduce the desired inequality (3.21).

\section{§4. Justifying THE ASYMPTOTICS}

1. Formal operations with discrepancies. At the beginning of the procedure of justifying the asymptotics, we do not take care of the smoothness properties of the problem data and the solution, as before.

The role of an approximate solution of problem (1.6) -1.8 will be played by the sum

$$
\mathcal{U}^{h}(x)=\mathbf{u}(x)+h X_{h}(x)\left(\mathcal{X}\left(x, h^{-1} x\right) \mathcal{D}\left(\nabla_{x}\right) \mathbf{u}(x)+U\left(x, h^{-1} x\right)\right),
$$

where $\mathbf{u}$ and $U$ are solutions of problems (1.36), (1.37), and (3.2), respectively, $\mathcal{X}$ is an asymptotic corrector satisfying (3.3) and (3.7), and $X_{h} \in C_{c}^{\infty}(\Omega)$ is a cutoff function equal to zero in the $(h \sqrt{n})$-neighborhood of $\partial \Omega$ and to 1 off the $(2 h \sqrt{n})$-neighborhood of $\partial \Omega$. The sizes of the neighborhoods are chosen so that if $\operatorname{supp} X_{h} \cap \varpi_{h}(\alpha) \neq \varnothing$, then the cell lies entirely in the perforated domain $\Omega(h)$. Also, we can ensure the inequalities

$$
0 \leq X_{h}(x) \leq 1, \quad\left|\nabla_{x}^{k} X_{h}(x)\right| \leq c_{k} h^{-k}, \quad k \in \mathbb{N} .
$$

Due to the cutoff function and the boundary condition (1.37), the vector-valued function $\mathcal{U}^{h}$ vanishes on the outer boundary $\Gamma(h)$. The requirements imposed on the problem data ensure that $\mathcal{U}^{h} \in H^{1}(\Omega(h))^{k}$. Thus, as a test function in the integral identity (1.11) we can take $\mathcal{T}^{h}=u^{h}-\mathcal{U}^{h} \in \stackrel{\circ}{H}^{1}(\Omega(h) ; \Gamma(h))^{k}$. Subtracting the form $a\left(\mathcal{U}^{h}, \mathcal{T}^{h} ; \Omega(h)\right)$ from both parts of the identity, we obtain

$$
a\left(\mathcal{T}^{h}, \mathcal{T}^{h} ; \Omega(h)\right)=\left(f^{h}, \mathcal{T}^{h}\right)_{\Omega(h)}+\left(g^{h}, \mathcal{T}^{h}\right)_{\Upsilon(h)}-\left(\mathcal{A D}\left(\nabla_{x}\right) \mathcal{U}^{h}, \mathcal{D}\left(\nabla_{x}\right) \mathcal{T}^{h}\right)_{\Omega(h)}
$$

By Theorem 1.1. the left-hand side of (4.3) majorizes the expression $c\left\|\mathcal{T}^{h} ; H^{1}(\Omega(h))\right\|^{2}$ with some positive constant $c$. Our nearest goal is to reshape the right-hand side of (4.3) to a form convenient for what follows.

Assuming that the cutoff function $X_{h}$ is a function of slow variables, we adopt the usual agreement about the simultaneous use of the two coordinate systems $x$ and $y$. We transform the last inner product in (4.3). We denote $T^{h}=X_{h} \mathcal{T}^{h}$ and, after commutation with the differential operator $\mathcal{D}\left(\nabla_{x}\right)$, transfer the cutoff function from the left position to the right one:

$$
\begin{aligned}
( & \left.\mathcal{A D} \mathcal{U}^{h}, \mathcal{D} \mathcal{T}^{h}\right)_{\Omega(h)}=\left(\mathcal{A}(\mathcal{D} \mathbf{u}+h \mathcal{D}(\mathcal{X D} \mathbf{u}+U)), \mathcal{D} T^{h}\right)_{\Omega(h)}+I_{X} \\
I_{X}= & h\left(\mathcal{D}\left(\nabla_{x} X_{h}\right)(\mathcal{X D} \mathbf{u}+U), \mathcal{A D} \mathcal{T}^{h}\right)_{\Omega(h)} \\
& -h\left(\mathcal{D}(\mathcal{X} \mathcal{D} \mathbf{u}+U), \mathcal{A D}\left(\nabla_{x} X_{h}\right)^{h}\right)_{\Omega(h)}+\left(\mathcal{D} \mathbf{u}, \mathcal{A D}\left(1-X_{h}\right) \mathcal{T}^{h}\right)_{\Omega(h)} .
\end{aligned}
$$


Note that $\mathcal{D}$ means the differential operator $\mathcal{D}\left(\nabla_{x}\right)$, and $\mathcal{D}\left(\nabla_{x} X_{h}\right)$ is a matrix-valued function.

Remark 4.1. Using relations (2.26) and (4.2) and the inequality $\rho_{h}(x) \leq c h$, valid on the support of the function $\left|\nabla_{x} X_{h}\right|$, we obtain

$$
\begin{aligned}
\left\|T^{h} ; H^{1}(\Omega(h))\right\| \leq & c\left(\left\|\nabla_{x} \mathcal{T}^{h} ; L_{2}(\Omega(h))\right\|\right. \\
& \left.\quad+h^{-1}\left\|\mathcal{T}^{h} ; L_{2}\left(\operatorname{supp}\left|\nabla_{x} X_{h}\right|\right)\right\|+\left\|\mathcal{T}^{h} ; L_{2}(\Omega(h))\right\|\right) \\
\leq c \| & \mathcal{T}^{h} ; H^{1}(\Omega(h)) \|,
\end{aligned}
$$

with a constant $c$ independent of $h \leq 1$ and $\mathcal{T}^{h} \in \stackrel{\circ}{H}^{1}(\Omega(h) ; \Gamma(h))^{k}$.

Now we consider the expressions that arise when the Green formula is applied:

$$
\begin{aligned}
& \left(\mathcal{A D}(\mathbf{u}+h(\mathcal{X D} \mathbf{u}+U)), \mathcal{D} T^{h}\right)_{\Omega(h)} \\
& \quad=\left(\mathcal{L}^{h}(\mathbf{u}+h(\mathcal{X} \mathcal{D} \mathbf{u}+U)), T^{h}\right)_{\Omega(h)}+\left(\mathcal{N}^{h}(\mathbf{u}+h(\mathcal{X} \mathcal{D} \mathbf{u}+U)), T^{h}\right)_{\Upsilon(h)} .
\end{aligned}
$$

We have

$$
\begin{aligned}
& \mathcal{L}^{h}\left(x, \nabla_{x}\right)\left(\mathbf{u}+h\left(\mathcal{X}\left(x, h^{-1} x\right) \mathcal{D}\left(\nabla_{x}\right) \mathbf{u}(x)+U\left(x, h^{-1} x\right)\right)\right) \\
&= h^{-1}\left(L^{1}\left(x, y, \nabla_{x}, \nabla_{y}\right) \mathbf{u}(x)+L^{0}\left(x, y, \nabla_{y}\right) \mathcal{X}(x, y) \mathcal{D}\left(\nabla_{x}\right) \mathbf{u}(x)\right) \\
&+h^{-1} L^{0}\left(x, y, \nabla_{y}\right) U(x, y)+h^{0}\left(L^{2}\left(x, y, \nabla_{x}\right) \mathbf{u}(x)\right. \\
&\left.\quad+{\overline{\mathcal{D}}\left(-\nabla_{x}\right)}^{\top} \mathcal{A}(x, y) \mathcal{D}\left(\nabla_{y}\right)\left(\mathcal{X}(x, y) \mathcal{D}\left(\nabla_{x}\right) \mathbf{u}(x)+U(x, y)\right)\right) \\
&+h^{1} \overline{\left(\mathcal{D}\left(-\nabla_{x}\right)+h^{-1} \mathcal{D}\left(-\nabla_{y}\right)\right)^{\top}} \mathcal{A}(x, y) \mathcal{D}\left(\nabla_{x}\right)\left(\mathcal{X}(x, y) \mathcal{D}\left(\nabla_{x}\right) \mathbf{u}(x)+U(x, y)\right) \\
&= h^{-1} F^{-1}+h^{-1} F^{U}+h^{0} F^{0}+h^{1} F^{1} .
\end{aligned}
$$

Similarly,

$$
\begin{aligned}
& \mathcal{N}^{h}\left(x, \nabla_{x}\right)\left(\mathbf{u}+h\left(\mathcal{X}\left(x, h^{-1} x\right) \mathcal{D}\left(\nabla_{x}\right) \mathbf{u}(x)+U\left(x, h^{-1} x\right)\right)\right) \\
&=h^{0}\left(N^{1}\left(x, y, \nabla_{x}\right) \mathbf{u}(x)+N^{0}\left(x, y, \nabla_{y}\right) \mathcal{X}(x, y) \mathcal{D}\left(\nabla_{x}\right) \mathbf{u}(x)\right) \\
& \quad+h^{0} N^{0}\left(x, y, \nabla_{y}\right) U(x, y)+h^{1} \overline{\mathcal{D}(\nu(y))} \mathcal{A}(x, y) \mathcal{D}\left(\nabla_{x}\right)\left(\mathcal{X}(x, y) \mathcal{D}\left(\nabla_{x}\right) \mathbf{u}(x)+U(x, y)\right) \\
&=: h^{0} G^{-1}+h^{0} G^{U}+h^{1} G^{1} .
\end{aligned}
$$

In accordance with formulas (3.2), (3.3) and (3.11)-(3.15), we find

$$
\begin{gathered}
F^{U}=F, \quad G^{U}=G, \quad F^{-1}=0, \quad G^{-1}=0, \\
\int_{\varpi} F^{0}(x, y) d y-\mathbf{f}^{0}(x)=0 .
\end{gathered}
$$

Also, the rule (3.1) shows that the sum $\mathcal{D}\left(\nabla_{x}\right)+h^{-1} \mathcal{D}\left(\nabla_{y}\right)$ coincides with the full differentiation operator $\mathcal{D}$. Consequently,

$$
\begin{aligned}
& h\left(F^{1}, T^{h}\right)_{\Omega(h)}+h\left(G^{1}, T^{h}\right)_{\Upsilon(h)}=h\left(\mathcal{Y}, \mathcal{D} T^{h}\right)_{\Omega(h)}=: I_{1}, \\
& \mathcal{Y}\left(x, h^{-1} x\right)=\left.\mathcal{A}(x, y) \mathcal{D}\left(\nabla_{x}\right)\left(\mathcal{X}(x, y) \mathcal{D}\left(\nabla_{x}\right) \mathbf{u}(x)+U(x, y)\right)\right|_{y=h^{-1} x} .
\end{aligned}
$$

Thus, formulas (1.38), (1.39), and (4.4) allow us to rewrite (4.3) in the form

$$
a\left(\mathcal{T}^{h}, \mathcal{T}^{h} ; \Omega(h)\right)=-I_{X}+I_{0}-I_{1}+\widetilde{I}+I^{\prime},
$$


where $I_{X}$ and $I_{1}$ are as defined in (4.5) and (4.8), respectively, and

$$
\begin{aligned}
I_{0} & =-\left(F^{0}, T^{h}\right)_{\Omega(h)}+\left(\operatorname{meas}_{n} \varpi\right)^{-1}\left(\mathbf{f}^{0}, T^{h}\right)_{\Omega(h)}, \\
\widetilde{I} & =\left(\tilde{f}^{h}, \mathcal{T}^{h}\right)_{\Omega(h)}+\left(\widetilde{g}^{h}, \mathcal{T}^{h}\right)_{\Upsilon(h)}, \\
I^{\prime} & =\left(h^{-1} F+\left(\operatorname{meas}_{n} \varpi\right)^{-1} \mathbf{f}^{0},\left(1-X_{h}\right) \mathcal{T}^{h}\right)_{\Omega(h)}+\left(G,\left(1-X_{h}\right) \mathcal{T}^{h}\right)_{\Upsilon(h)} .
\end{aligned}
$$

2. Auxiliary estimates. To handle the expression (4.10), we need two simple and, to some extent, known facts.

Lemma 4.1. Suppose $t \in H^{1}(\Omega(h))$ and $t(x)=0$ for $\operatorname{dist}(x, \partial \Omega) \leq h \sqrt{n}$. Suppose also that the functions $x \mapsto v(x)$ and $x \mapsto z\left(x, h^{-1} x\right)$ satisfy the relations in one of the following two groups:

$$
v(x)=1, z \in H^{\delta}\left(\Omega ; L_{\infty}(\varpi)\right) \quad \text { or } \quad v \in C^{0, \delta}(\Omega), z(x, y)=z(x), z \in L_{2}(\varpi),
$$

where $\delta \in(0,1)$ and

$$
\int_{\varpi} z(x, y) d y=0 \quad \text { for a.e. } \quad x \in \Omega \text {. }
$$

Then

$$
\left|(v z, t)_{\Omega(h)}\right| \leq c h^{\delta}\left\|v ; C^{0, \delta}(\Omega)\right\|\|z\|\left\|t ; H^{1}(\Omega(h))\right\|,
$$

where $\|z\|$ is any of the norms in the spaces occurring in (4.13).

Proof. The condition imposed on $t$ implies that this function vanishes on all cells $\varpi_{h}(\alpha)$ that do not lie entirely in the perforated domain $\Omega(h)$. The summation over all cells $\varpi_{h}(\alpha) \subset \Omega(h)$ will be denoted by $\sum_{\alpha}$.

First, we suppose that the formulas of the first group in (4.13) are fulfilled; in particular, $v=1$. We have

$$
\begin{aligned}
(z, t)_{\Omega(h)}= & \sum_{\alpha} \int_{\varpi_{h}(\alpha)} \overline{t(x)} z\left(x, \frac{x}{h}\right) d x \\
= & \sum_{\alpha}\left(\int_{\varpi_{h}(\alpha)} \overline{\left(t(x)-t_{\alpha}\right)} z\left(x, \frac{x}{h}\right) d x\right. \\
& \left.\quad+h^{-n} \overline{t_{\alpha}}\left(\operatorname{meas}_{n} \varpi\right)^{-1} \int_{\varpi_{h}(\alpha)} \int_{\varpi_{h}(\alpha)}\left(z\left(x, \frac{x}{h}\right)-z\left(\mathbf{x}, \frac{x}{h}\right)\right) d x d \mathbf{x}\right) .
\end{aligned}
$$

Here $t_{\alpha}$ denotes the mean value of $t$ over the cell,

$$
t_{\alpha}=h^{-n}\left(\operatorname{meas}_{n} \varpi\right)^{-1} \int_{\varpi_{h}(\alpha)} t(x) d x, \quad\left|t_{\alpha}\right|^{2} \leq h^{-n}\left(\operatorname{meas}_{n} \varpi\right)^{-1}\left\|t ; L_{2}\left(\varpi_{h}(\alpha)\right)\right\|^{2} .
$$

Observe that the subtrahend $z\left(\mathbf{x}, h^{-1} x\right)$ was introduced in the last integrand; the requirement (4.14) shows that this is indeed possible. By the Poincaré inequality (see the comments on relations (2.18) and (2.22) $)$, we get

$$
\left\|t-t_{\alpha} ; L_{2}\left(\varpi_{h}^{\bullet}(\alpha)\right)\right\|^{2} \leq c h^{2}\left\|\nabla_{x} t ; L_{2}\left(\varpi_{h}^{\bullet}(\alpha)\right)\right\|^{2}
$$


(recall that $\varpi^{\bullet}$ stands for the extended periodicity cell). We continue transformations:

$$
\begin{aligned}
\left(\int_{\varpi_{h}(\alpha)}\right. & \left.\int_{\varpi_{h}(\alpha)}\left(z\left(x, \frac{x}{h}\right)-z\left(\mathbf{x}, \frac{x}{h}\right)\right) d x d \mathbf{x}\right)^{2} \\
\leq & \left(\operatorname{meas}_{n} \varpi_{h}\right)^{2} \sup _{x, \mathbf{x} \in \varpi_{h}(\alpha)}|x-\mathbf{x}|^{n+2 \delta} \\
& \times \int_{\varpi_{h}(\alpha)} \int_{\varpi_{h}(\alpha)} \sup _{y \in \varpi} \mid\left(z(x, y)-\left.z(\mathbf{x}, y)\right|^{2} \frac{d x d \mathbf{x}}{|x-\mathbf{x}|^{n+2 \delta}}\right. \\
\leq & c h^{3 n+2 \delta} \int_{\varpi_{h}(\alpha)} \int_{\varpi_{h}(\alpha)}\left\|z(x, \cdot)-z(\mathbf{x}, \cdot) ; L_{\infty}(\varpi)\right\|^{2} \frac{d x d \mathbf{x}}{|x-\mathbf{x}|^{n+2 \delta}} \\
= & : h^{3 n+2 \delta} J_{h}(\alpha) .
\end{aligned}
$$

We employ the Cauchy inequality with an arbitrary factor $\varepsilon_{i}>0$ and use the above estimates to obtain

$$
\begin{aligned}
\left|(z, t)_{\Omega(h)}\right| \leq c \sum_{\alpha}\left(\varepsilon_{1} h^{2}\left\|\nabla_{x} t ; L_{2}\left(\varpi_{h}^{*}(\alpha)\right)\right\|^{2}\right. & +\frac{1}{\varepsilon_{1}}\left\|z ; L_{2}\left(\varpi_{h}(\alpha) ; L_{\infty}(\varpi)\right)\right\|^{2} \\
& \left.+\varepsilon_{2} h^{n}\left|t_{\alpha}\right|^{2}+\frac{1}{\varepsilon_{2}} h^{-3 n} h^{3 n+2 \delta} J_{h}(\alpha)\right) \\
\leq c( & \varepsilon_{1} h^{2}\left\|\nabla_{x} t ; L_{2}(\Omega(h))\right\|^{2}+\frac{1}{\varepsilon_{1}}\left\|z ; L_{2}\left(\Omega(h) ; L_{\infty}(\varpi)\right)\right\|^{2} \\
& \left.+\varepsilon_{2}\left(\operatorname{meas}_{n} \varpi\right)^{-1}\left\|t ; L_{2}(\Omega(h))\right\|^{2}+\frac{1}{\varepsilon_{2}} h^{2 \delta}\left\|z ; H^{\delta}\left(\Omega ; L_{\infty}(\varpi)\right)\right\|^{2}\right) .
\end{aligned}
$$

Since the cases where $t=0$ or $z=0$ are not interesting (the claim becomes trivial), we put

$$
\begin{aligned}
& \varepsilon_{1}=h^{-1}\left\|\nabla_{x} t ; L_{2}(\Omega(h))\right\|^{-1}\left\|z ; L_{2}\left(\Omega(h) ; L_{\infty}(\varpi)\right)\right\|, \\
& \varepsilon_{2}=h^{\delta}\left\|t ; L_{2}(\Omega(h))\right\|^{-1}\left\|z ; H^{\delta}\left(\Omega ; L_{\infty}(\varpi)\right)\right\|,
\end{aligned}
$$

arriving at the desired relation (4.15).

Now, suppose that the formulas of the second group in (4.13) are fulfilled. Doing transformations completely similar to those above and using (4.14), we obtain

$$
\begin{aligned}
(v z, t)_{\Omega(h)}= & \sum_{\alpha}\left(v(h \alpha) \int_{\varpi_{h}(\alpha)} \overline{\left(t(x)-t_{\alpha}\right)} z\left(\frac{x}{h}\right) d x\right. \\
& \quad+\int_{\varpi_{h}(\alpha)}\left(v(x)-v(h \alpha) \overline{t(x)} z\left(\frac{x}{h}\right) d x\right) \\
= & : \sum_{\alpha}\left(J_{h}^{1}(\alpha)+J_{h}^{2}(\alpha)\right) .
\end{aligned}
$$

Note that $h \alpha$ is one of the vertices of the cube $\mathbb{Q}_{h}(\alpha) \supset \varpi_{h}(\alpha)$. The Poincaré inequality (4.16) implies that

$$
\begin{aligned}
\left|J_{h}^{1}(\alpha)\right| & \leq c|v(h \alpha)| h\left\|\nabla_{x} t ; L_{2}\left(\varpi_{h}(\alpha)\right)\right\| h^{n / 2}\left\|z ; L_{2}(\varpi)\right\| \\
& \leq c\left\|v ; L_{\infty}(\Omega)\right\|\left(\varepsilon_{1}\left\|\nabla_{x} t ; L_{2}\left(\varpi_{h}(\alpha)\right)\right\|^{2}+\frac{1}{\varepsilon_{1}} h^{2+n}\left\|z ; L_{2}(\varpi)\right\|^{2}\right) .
\end{aligned}
$$

The clear relation $|v(x)-v(h \alpha)| \leq c h^{\delta}\left\|v ; C^{0, \delta}(\Omega)\right\|$ entails the formula

$$
\left|J_{h}^{2}(\alpha)\right| \leq \operatorname{ch}^{\delta}\left\|v ; C^{0, \delta}(\Omega)\right\|\left(\varepsilon_{2}\left\|t ; L_{2}\left(\varpi_{h}(\alpha)\right)\right\|^{2}+\frac{1}{\varepsilon_{2}} h^{n}\left\|z ; L_{2}(\varpi)\right\|^{2}\right) .
$$


Observe that the number of cells $\varpi_{h}(\alpha)$ in the domain $\Omega(h)$ is at most $h^{-n} \operatorname{meas}_{n} \Omega$. Therefore, collecting relations (4.19), (4.20) and performing the summation in (4.18), we find

$$
\left|(v z, t)_{\Omega(h)}\right| \leq c\left\|v ; C^{0, \delta}(\Omega)\right\|\left(\left(\varepsilon_{1}+h^{\delta} \varepsilon_{2}\right)\left\|t ; H^{1}(\Omega(h))\right\|^{2}+\left(\varepsilon_{1}^{-1} h^{2}+h^{\delta} \varepsilon_{2}^{-1}\right)\left\|z ; L_{2}(\varpi)\right\|^{2}\right) .
$$

To complete the proof, it now suffices to repeat what was said before formula (4.17) and put

$$
\varepsilon_{1}=h \varepsilon_{2}=h\left\|t ; H^{1}(\Omega(h))\right\|^{-1}\left\|z ; L_{2}(\varpi)\right\| .
$$

3. Justification of the asymptotics in the case of higher smoothness relative to the fast variables. Suppose that conditions (1.46)-(1.48) are satisfied. By Proposition 3.2 (2), for the solution of problem (3.2), 3.6 we have

$$
U \in H^{1+\delta}\left(\Omega ; C_{\text {per }}^{2, \delta_{1}}(\varpi)\right)^{k}, \quad\left\|U ; H^{1+\delta}\left(\Omega ; C_{\text {per }}^{2, \delta_{1}}(\varpi)\right)\right\| \leq c \mathbf{n}_{H} .
$$

A similar statement in which the Sobolev spaces are replaced with Hölder ones (for dependences on the slow variables $x$ ) yields

$$
\mathcal{X} \in C^{1, \delta_{A}}\left(\Omega ; C_{\text {per }}^{2, \delta_{1}}(\varpi)\right)^{k \times N}
$$

for the solution of problem (3.3), (3.7). Also, formulas (3.15) and (3.13) show that (1.49) is true and that

$$
\mathbf{F} \in H^{1+\delta}(\Omega)^{N}, \quad \mathbf{f} \in H^{\delta}(\Omega)^{k}, \quad\left\|\mathbf{F} ; H^{1+\delta}(\Omega)\right\|+\left\|\mathbf{f} ; H^{\delta}(\Omega)\right\| \leq c \mathbf{n}_{H} .
$$

Consequently, by Proposition 3.1 .

$$
\mathbf{u} \in H^{2+\delta}(\Omega)^{k}, \quad\left\|\mathbf{u} ; H^{2+\delta}(\Omega)\right\| \leq c \mathbf{n}_{H} .
$$

We recall that $\mathbf{n}_{H}$ is the sum of the norms of the vector-valued functions (1.47) in the spaces indicated. Clearly, relations (4.21) and (1.48) imply that the functions $x \mapsto$ $U\left(x, h^{-1} x\right)$ and $x \mapsto \mathcal{X}\left(x, h^{-1} x\right) \mathcal{D}\left(\nabla_{x}\right) \mathbf{u}(x)$ belong to the Sobolev space $H^{1}(\Omega(h))^{k}$; i.e., the asymptotic approximation (4.1) to the solution $u^{h}(x)$ of problem (1.6)-(1.8) indeed falls into the space $\stackrel{\circ}{H}^{1}(\Omega ; \Gamma(h))^{k}$.

We estimate the terms on the right in (4.9). The supports of the vector-valued functions $\mathcal{D}\left(\nabla_{x} X_{h}\right) \mathcal{T}^{h}$ and $\left(1-X_{h}\right) \mathcal{T}^{h}$ are included in the set $\overline{\Theta(h)}$ occurring in Lemma 2.1 . Therefore,

$$
\begin{aligned}
\left|I_{X}\right| \leq \operatorname{ch}( & h^{-1}\left\|\mathcal{X} \mathcal{D} \mathbf{u}+U ; L_{2}(\Theta(h))\right\|\left\|\mathcal{T}^{h} ; H^{1}(\Theta(h))\right\| \\
& +\left\|\mathcal{D}(\mathcal{X} \mathcal{D} \mathbf{u}+U) ; L_{2}(\Theta(h))\right\| h^{-1}\left\|\mathcal{T}^{h} ; L_{2}(\Theta(h))\right\| \\
& \left.\quad+h^{-1}\left\|\mathcal{D} \mathbf{u} ; L_{2}\left(\theta_{h}\right)\right\|\left(h^{-1}\left\|\mathcal{T}^{h} ; L_{2}(\Theta(h))\right\|+\left\|\nabla_{x} \mathcal{T}^{h} ; L_{2}(\Theta(h))\right\|\right)\right) \\
& \leq c h\left(h^{-1}\left(h^{1 / 2}+h^{1 / 2}\right)+\left(1+h^{-1} h^{1 / 2}\right)+h^{-1} h^{1 / 2}\right) \mathbf{n}_{H}\left\|\mathcal{T}^{h} ; H^{1}(\Omega(h))\right\| \\
\leq & c h^{1 / 2} \mathbf{n}_{H}\left\|\mathcal{T}^{h} ; H^{1}(\Omega(h))\right\| .
\end{aligned}
$$

These calculations need an explanation. To the vector-valued functions $\mathcal{T}^{h}$ and $\mathcal{D} \mathbf{u}$ we have applied the inequalities (2.25) and (2.24), respectively. Also, we have used the estimates

$$
\begin{aligned}
\left\|\mathcal{X D} \mathbf{D} ; L_{2}(\Theta(h))\right\| & \leq c_{\mathcal{X}}\left\|\nabla_{x} \mathbf{u} ; L_{2}(\Theta(h))\right\|, \\
h\left\|\mathcal{D} \mathcal{X} \mathcal{D} \mathbf{u} ; L_{2}(\Theta(h))\right\| & \leq C_{\mathcal{X}}\left(h\left\|\nabla_{x}^{2} \mathbf{u} ; L_{2}(\Theta(h))\right\|+\left\|\nabla_{x} \mathbf{u} ; L_{2}(\Theta(h))\right\|\right) .
\end{aligned}
$$

Finally, the terms involving $U$ were handled with the help of Lemma 2.3 with $z=U$ and $z=\mathcal{D}\left(\nabla_{y}\right) U$, and

$$
\begin{aligned}
h\left\|\mathcal{D} U ; L_{2}(\Theta(h))\right\| & \leq c\left(\left\|\mathcal{D}\left(\nabla_{y}\right) U ; L_{2}(\Theta(h))\right\|+h\left\|\mathcal{D}\left(\nabla_{x}\right) U ; L_{2}(\Theta(h))\right\|\right) \\
& \leq c\left(h^{1 / 2}\left\|U ; H^{1}\left(\Omega ; H_{\infty}^{1}(\varpi)\right)\right\|+h\left\|U ; H^{1}\left(\Omega ; L_{\infty}(\varpi)\right)\right\|\right) .
\end{aligned}
$$


Similar arguments involving relations (1.47), the trace inequality (2.31), and estimate (4.6) yield the following inequality for the quantity (4.12):

$$
\left|I^{\prime}\right| \leq c\left(\left(h^{-1} h^{1 / 2}+1\right) h+h^{1 / 2}\right) \mathbf{n}_{H}\left\|\mathcal{T}^{h} ; H^{1}(\Omega(h))\right\| .
$$

By formulas (1.48), (4.22), and (4.23), the matrix-valued functions

$$
\Omega(h) \ni x \mapsto \mathcal{A}\left(x, h^{-1} x\right), \quad x \mapsto \mathcal{X}\left(x, h^{-1} x\right),\left.\quad x \mapsto \mathcal{D}\left(\nabla_{x}\right) \mathcal{X}(x, y)\right|_{y=h^{-1} x}
$$

are bounded, and we have $\mathbf{u} \in H^{2}(\Omega)^{k} \subset H^{2}(\Omega(h))^{k}$, so that, by (4.8),

$$
\begin{aligned}
\left|I_{1}\right| & \leq \operatorname{ch}\left(\left\|\mathbf{u} ; H^{2}(\Omega)\right\|+\left\|U ; H^{1}\left(\Omega ; L_{\infty}(\varpi)\right)\right\|\right)\left\|\mathcal{T}^{h} ; H^{1}(\Omega(h))\right\| \\
& \leq \operatorname{ch} \mathbf{n}_{H}\left\|\mathcal{T}^{h} ; H^{1}(\Omega(h))\right\| .
\end{aligned}
$$

The definition (1.43) shows that

$$
|\widetilde{I}| \leq c h^{\delta} \widetilde{\mathbf{n}}\left\|\mathcal{T}^{h} ; H^{1}(\Omega(h))\right\|
$$

It remains to consider the quantity (4.10), which can be represented in the form

$$
\begin{aligned}
& \int_{\Omega(h)}{\overline{T^{h}(x)}}^{\top}\left(Z_{(\mathbf{u})}^{0}\left(x, \frac{x}{h}\right)+Z_{(\mathbf{u})}^{1}\left(x, \frac{x}{h}\right) \nabla_{x}\right) \mathcal{D}\left(\nabla_{x}\right) \mathbf{u}(x) d x \\
& \quad+\int_{\Omega(h)}{\overline{T^{h}(x)}}^{\top}\left(Z_{(U)}^{0}\left(x, \frac{x}{h}\right)+Z_{(U)}^{1}\left(x, \frac{x}{h}\right) \nabla_{x}\right) \mathcal{D}\left(\nabla_{y}\right) U\left(x, \frac{x}{h}\right) d x,
\end{aligned}
$$

where $Z_{(\mathbf{u})}^{i}$ and $Z_{(U)}^{i}$ are matrix-valued functions of appropriate sizes with entries of class $C^{0, \delta_{A}}\left(\Omega ; C_{\mathrm{per}}^{0, \delta_{1}}(\varpi)\right)$ that satisfy (4.14) (see the orthogonality condition (4.7)). Since $\mathcal{D}\left(\nabla_{x}\right) \mathbf{u} \in H^{1+\delta}(\Omega)^{N}, \mathcal{D}\left(\nabla_{y}\right) U \in H^{1+\delta}\left(\Omega ; C_{\text {per }}^{1, \delta_{1}}(\varpi)\right)^{N}$, and $\delta_{A}>\delta$, we have

$$
z:=\left(Z_{(\mathbf{u})}^{0}+Z_{(\mathbf{u})}^{1} \nabla_{x}\right) \mathcal{D}\left(\nabla_{x}\right) \mathbf{u}+\left(Z_{(U)}^{0}+Z_{(U)}^{1} \nabla_{x}\right) \mathcal{D}\left(\nabla_{y}\right) U \in H^{\delta}\left(\Omega ; L_{\infty}(\varpi)\right)^{k} .
$$

Consequently, the requirements of Lemma 4.1 (the first group of relations in (4.13)) are fulfilled, which implies the estimate

$$
\left|I_{0}\right| \leq c h^{\delta} \mathbf{n}_{H}\left\|\mathcal{T}^{h} ; H^{1}(\Omega(h))\right\| .
$$

Now we want to deduce the desired inequality (1.50) in Theorem 1.2 from formulas (4.24), (4.27)-(4.30). For this, we need to remove the cutoff function $X_{h}$ from the (intermediate) asymptotic approximation, i.e., to estimate the norm

$$
\left\|h\left(1-X_{h}\right)(\mathcal{X} \mathcal{D} \mathbf{u}+U) ; H^{1}(\Omega(h))\right\|=\left\|h\left(1-X_{h}\right)(\mathcal{X} \mathcal{D} \mathbf{u}+U) ; H^{1}(\Theta(h))\right\| .
$$

This can easily be done with the help of the already used relations (4.25) and (4.26); the result shows that the norm (4.31) does not exceed $c h^{1 / 2} \mathbf{n}_{H}$. Finally, the first statement in Corollary 1.1 is ensured by the following simple observation: the expressions

$$
h\left\|\mathcal{X} \mathcal{D} \mathbf{u}+U ; L_{2}(\Omega(h))\right\|, \quad h\left\|\left.\nabla_{x}(\mathcal{X}(\cdot, y) \mathcal{D} \mathbf{u}+U(\cdot, y))\right|_{y=h^{-1} x} ; L_{2}(\Omega(h))\right\|
$$

admit one and the same majorant $c h^{1 / 2} \mathbf{n}_{H}$.

So, the claims stated in Subsection 5 of $\S 1$ are proved in the case where the requirements (1.47) and (1.48) are fulfilled. 
4. Justification of the asymptotics in the case of higher smoothness relative to the slow variables. Now we assume conditions (1.46) and (1.51). Then, by Propositions 3.2(1) and 3.1, we get

$$
\begin{aligned}
\mathcal{X}(x, y) & =\mathcal{X}(y), \quad \mathcal{X} \in H_{\mathrm{per}}^{1}(\varpi)^{k \times N}, \\
U(x, y) & =\mathbf{a}(x) U^{\prime}(y), \quad U^{\prime} \in H_{\mathrm{per}}^{1}(\varpi)^{k}, \\
\mathbf{f} & \in C^{0, \delta}(\Omega)^{k}, \quad \mathbf{u} \in C^{2, \delta}(\Omega)^{k},
\end{aligned}
$$

the norms of the vector-valued functions are dominated by the quantity (1.55), and the asymptotic approximation (4.1) belongs to the Sobolev space $\stackrel{\circ}{H}^{1}(\Omega ; \Gamma(h))^{k}$.

Since the number of the cells $\varpi_{h}(\alpha)$ that intersect the narrow strip $\Theta(h)$ is at most $c h^{1-n}$, we see that

$$
\begin{aligned}
\left\|\mathcal{X} ; L_{2}(\Theta(h))\right\| & \leq c h^{1 / 2}\left\|\mathcal{X} ; L_{2}(\varpi)\right\|, \\
\left\|U^{\prime} ; L_{2}(\Theta(h))\right\| & \leq c h^{1 / 2}\left\|U^{\prime} ; L_{2}(\varpi)\right\| .
\end{aligned}
$$

Now we use Remark 4.1 and the fact that the function a, the entries of the matrix $\left|\mathcal{D}\left(\nabla_{x}\right) \mathbf{u}\right|$, and their derivatives can be estimated pointwise (see (1.52) and (4.35)). Employing relations (4.33), (4.34), and (4.36), we get

$$
\begin{gathered}
\left|I_{X}\right| \leq \operatorname{ch}\left(h^{-1}\left\|\mathcal{X} \mathcal{D}\left(\nabla_{x}\right) \mathbf{u}+\mathbf{a} U^{\prime} ; L_{2}(\Theta(h))\right\|\right. \\
\quad+\left\|\mathcal{D}\left(\nabla_{x}\right)\left(\mathcal{X} \mathcal{D}\left(\nabla_{x}\right) \mathbf{u}+\mathbf{a} U^{\prime}\right) ; L_{2}(\Theta(h))\right\| \\
\left.\quad+h^{-1}\left\|\mathcal{D}\left(\nabla_{x}\right) \mathbf{u} ; L_{2}(\Theta(h))\right\|\right)\left\|\mathcal{T}^{h} ; H^{1}(\Omega(h))\right\| \\
\leq \operatorname{ch}\left(h^{-1}\left(\left\|\mathbf{u} ; H_{\infty}^{1}(\Omega)\right\|\left\|\mathcal{X} ; L_{2}(\Theta(h))\right\|+\left\|\mathbf{a} ; L_{\infty}(\Omega)\right\|\left\|U^{\prime} ; L_{2}(\Theta(h))\right\|\right)\right. \\
\quad+\left(\left\|\mathbf{u} ; H_{\infty}^{2}(\Omega)\right\|\left\|\mathcal{X} ; L_{2}(\Theta(h))\right\|+\left\|\mathbf{u} ; H_{\infty}^{1}(\Omega)\right\|\left\|\nabla_{x} \mathcal{X} ; L_{2}(\Theta(h))\right\|\right. \\
\left.\quad+\left\|\mathbf{a} ; H_{\infty}^{1}(\Omega)\right\|\left\|U^{\prime} ; L_{2}(\Theta(h))\right\|+\left\|\mathbf{a} ; L_{\infty}(\Omega)\right\|\left\|\nabla_{x} U^{\prime} ; L_{2}(\Theta(h))\right\|\right) \\
\left.\quad+h^{-1}\left\|\mathbf{u} ; H_{\infty}^{1}(\Omega)\right\|\left(\operatorname{meas}_{n} \Theta(h)\right)^{1 / 2}\right)\left\|\mathcal{T}^{h} ; H^{1}(\Omega(h))\right\| \\
\leq c h^{1 / 2} \mathbf{n}_{C}\left\|\mathcal{T}^{h} ; H^{1}(\Omega(h))\right\| .
\end{gathered}
$$

For similar reasons, we have

$$
\left|I^{\prime}\right| \leq c h^{1 / 2} \mathbf{n}_{C}\left\|\mathcal{T}^{h} ; H^{1}(\Omega(h))\right\| .
$$

Estimate (4.29) of the quantity (4.11) can be taken from the preceding subsection, and the estimate

$$
\left|I_{1}\right| \leq \operatorname{ch}^{1} \mathbf{n}_{C}\left\|\mathcal{T}^{h} ; H^{1}(\Omega(h))\right\|
$$

for the quantity defined in (4.8) becomes obvious, because the maximum of the modulus of the factors $\mathcal{A}\left(h^{-1} x\right), \mathcal{D}\left(\nabla_{x}\right) \mathbf{u}(x), \mathbf{a}(x)$ and $\nabla_{x}^{2} \mathbf{u}(x), \nabla_{x} \mathbf{a}(x)$ can be majorized.

Finally, we note that the orthogonality condition (4.7) allows us to rewrite (4.10) in the form

$$
\int_{\Omega}{\overline{T^{h}(x)}}^{\top}\left(z^{1}\left(h^{-1} x\right) v^{1}(x)+z^{2}\left(h^{-1} x\right) v^{2}(x)\right) d x,
$$

where $v^{1}(x)=\nabla_{x}^{2} \mathbf{u}(x), v^{2}(x)=\nabla_{x} \mathbf{a}(x)$, while $z^{1}\left(h^{-1} x\right)$ and $z^{2}\left(h^{-1} x\right)$ are matrices of appropriate sizes, which have zero mean over the cell $\varpi$ and satisfy

$$
\left\|z^{1} ; L_{2}(\varpi)\right\| \leq c\left\|\mathcal{A} ; L_{2}(\varpi)\right\|, \quad\left\|z^{2} ; L_{2}(\varpi)\right\| \leq c\left\|\mathcal{A} ; L_{\infty}(\varpi)\right\|\left\|U^{\prime} ; L_{2}(\varpi)\right\| .
$$

Consequently, all conditions of Lemma 4.1 are fulfilled. By Remark 4.1 this implies the estimate

$$
\left|I_{0}\right| \leq \operatorname{ch}^{\delta} \mathbf{n}_{C}\left\|\mathcal{T}^{h} ; H^{1}(\Omega(h))\right\| .
$$


Formulas (4.37)-(4.40) and (4.29) complete the proof of Theorem 1.3 and of the second part of Corollary 1.1] because the majorant $c h^{1 / 2} \mathbf{n}_{C}$ for the norms (4.31) and (4.32) can also be obtained with the help of the above arguments.

The authors are deeply grateful to T. A. Suslina for valuable discussions of the subject, a careful reading of the manuscript, and help in eliminating misprints.

\section{REFERENCES}

[1] O. A. Ladyzhenskaya, Boundary value problems of mathematical physics, Nauka, Moscow, 1973; English transl., Appl. Math. Sci., vol. 49, Springer-Verlag, New York, 1985. MR0599579 (58:29032) MR0793735 (87f:35001)

[2] J. Nečas, Les méthodes directes en théorie des équations elliptiques, Masson, Paris; Academia, Prague, 1967. MR0227584 (37:3168)

[3] S. A. Nazarov, Self-adjoint elliptic boundary-value problems. The polynomial property and formally positive operators, Probl. Mat. Anal., No. 16, S.-Peterburg. Univ., St. Petersburg, 1997, pp. 167-192; English transl., J. Math. Sci. (New York) 92 (1998), no. 6, 4338-4353. MR1668418(2000a:35042)

[4] _ Polynomial property of selfadjoint elliptic boundary value problems, and the algebraic description of their attributes, Uspekhi Mat. Nauk 54 (1999), no. 5, 77-142; English transl., Russian Math. Surveys 54 (1999), no. 5, 947-1014. MR1741662 (2001k:35073)

[5] S. G. Lekhnit'skiǔ, Theory of elasticity of an anisotropic body, 2nd ed., Nauka, Moscow, 1977. (Russian) MR0502604 (58:19575)

[6] S. A. Nazarov, Asymptotic theory of thin plates and rods. Dimension reduction and integral estimates. Vol. 1, Nauchn. Kniga, Novosibirsk, 2002. (Russian)

[7] V. Z. Parton and B. A. Kudryavtsev, Electro-elasticity of piezoelectric and electroconductive bodies, Nauka, Moscow, 1988. (Russian)

[8] S. A. Nazarov, Uniform estimates of remainders in asymptotic expansions of solutions to the problem on eigenoscillations of a piezoelectric plate, Probl. Mat. Anal., No. 25, Tamara Rozhkovskaya, Novosibirsk, 2003, pp. 99-188; English transl., J. Math. Sci. (New York) 114 (2003), no. 5, 16571725. MR 1981301 (2004e:35028)

[9] E. A. Akimova, S. A. Nazarov, and G. A. Chechkin, The weighted Korn inequality: the "tetris" procedure that serves an arbitrary periodic plate, Dokl. Akad. Nauk 380 (2001), no. 4, 439-442. (Russian) MR 1875497

[10] _ Asymptotics of the solution of the problem of the deformation of an arbitrary locally periodic thin plate, Tr. Mosk. Mat. Obshch. 65 (2004), 3-34; English transl., Trans. Moscow Math. Soc. 2004, 1-29. MR2193435 (2006j:74056)

[11] S. A. Nazarov, Korn's inequalities for elastic joints of massive bodies, thin plates, and rods, Uspekhi Mat. Nauk 63 (2008), no. 1, 37-110; English transl., Russian Math. Surveys 63 (2008), no. 1, 35107. MR2406182 (2009c:35457)

[12] _ Justification of the asymptotic theory of thin rods. Integral and pointwise estimates, Probl. Mat. Anal., No. 17, S.-Peterburg. Univ., St. Petersburg, 1997, pp. 101-152; English transl., J. Math. Sci (New York) 97 (1999), no. 4, 4245-4279. MR1788230(2002d:74045)

[13] A. Bensoussan, J.-L. Lions, and G. Papanicolaou, Asymptotic analysis for periodic structures, Stud. in Math. Appl., vol. 5, North-Holland, Amsterdam-New York, 1978. MR0503330 (82h:35001)

[14] N. S. Bakhvalov and G. P. Panasenko, Homogenization: Averaging processes in periodic media, Mathematical Problems of the Mechanics of Composite Materials, Nauka, Moscow, 1984; English transl., Math. Appl. (Soviet Ser.), vol. 36, Kluwer Acad. Publ. Group, Dordrecht, 1989. MR0797571 (86m:73049) MR:1112788 (92d:73002)

[15] E. Sanchez-Palencia, Nonhomogeneous media and vibration theory, Lecture Notes in Phys., vol. 127, Springer-Verlag, Berlin-New York, 1980. MR0578345 (82j:35010)

[16] O. A. Oleŭnik, G. A. Iosif'yan, and A. S. Shamaev, Mathematical problems in the theory of strongly inhomogeneous elastic media, Moskov. Gos. Univ., Moscow, 1990. (Russian) MR1115306 (92i:73009)

[17] S. A. Nazarov, Homogenization of elliptic systems with periodic coefficients: Weigted $L^{p}$ and $L^{\infty}$ estimates for asymptotic remainders, Algebra i Analiz 18 (2006), no. 2, 117-166; English transl., St. Petersburg Math. J. 18 (2007), no. 2, 269-304. MR2244938(2007d:35016)

[18] O. A. Ladyzhenskaya and N. N. Ural'tseva, Linear and quasilinear equations of elliptic type, 2nd ed., Nauka, Moscow, 1973; English transl. of 1st ed., Acad. Press, New York-London, 1968. MR0509265 (58:23009) MR0244627(39:5941) 
[19] M. Sh. Birman and T. A. Suslina, Second order periodic differential operators. Threshold properties and homogenization, Algebra i Analiz 15 (2003), no. 5, 1-108; English transl., St. Petersburg Math. J. 15 (2004), no. 5, 639-714. MR2068790 (2005k:47097)

[20] _ Homogenization with corrector term for periodic elliptic differential operators, Algebra $\mathrm{i}$ Analiz 17 (2005), no. 6, 1-104; English transl., St. Petersburg Math. J. 17 (2006), no. 6, 897-973. MR.2202045 (2006k:35011)

[21] Homogenization with corrector term for periodic differential operators. Approximation of solutions in the Sobolev class $H^{1}\left(\mathbb{R}^{d}\right)$, Algebra i Analiz 18 (2006), no. 6, 1-130; English transl., St. Petersburg Math. J. 18 (2007), no. 6, 857-955. MR2307356 (2008d:35008)

[22] V. V. Zhikov, On the spectral method in homogenization theory, Tr. Mat. Inst. Steklov. 250 (2005), 95-104; English transl., Proc. Steklov Inst. Math. 2005, no. 3 (250), 85-94. MR2200910 (2006i:35016)

[23] V. V. Zhikov and S. E. Pastukhova, On operator estimates for some problems in homogenization theory, Russ. J. Math. Phys. 12 (2005), no. 4, 515-524. (English) MR2201316 (2007c:35014)

[24] S. E. Pastukhova, On some estimates from the homogenization of problems in plasticity theory, Dokl. Akad. Nauk 406 (2006), no. 5, 604-608. (Russian) MR2347320 (2008d:35016)

[25] S. Agmon, A. Douglis, and L. Nirenberg, Estimates near the boundary for solutions of elliptic partial differential equations satisfying general boundary conditions. II, Comm. Pure Appl. Math. 17 (1964), 35-92. MR0162050(28:5252)

[26] V. A. Solonnikov, General boundary value problems for systems elliptic in the sense of A. Douglis and L. Nirenberg. I, Izv. Akad. Nauk SSSR Ser. Mat. 28 (1964), 665-706. (Russian); II, Tr. Mat. Inst. Steklov. 92 (1966), 233-297; English transl. in Proc. Steklov Inst. Math. 1968. MR0211070 (35:1952) MR0211071 (35:1953) ITALY

Department of Engineering, University of Sannio, Corso Garibaldi, 107, 84100 Benevento,

E-mail address: giuseppe.cardone@unisannio.it

Department of Automation, Electromagnetism, Information and Industrial Mathematics, University of Cassino, Via G. Di Biasio, 43, 03043 Cassino (FR), Italy

E-mail address: corbo@unicas.it

Institute of Mechanical Engineering Problems, Bol'shoi Prospekt V.O. 61, St. Petersburg 199178, RUSSIA

E-mail address: srgnazarov@yahoo.co.uk

Received 24/NOV/2008

Translated by A. PLOTKIN 